\title{
Axonal Guidance Defects in a Caenorhabditis elegans Mutant Reveal Cell-extrinsic Determinants of Neuronal Morphology
}

\author{
Siegfried Hekimi' and Douglas Kershaw ${ }^{2}$ \\ 'Department of Biology, McGill University, Montréal, Quebec, Canada H3A 1B1 and ${ }^{2}$ Medical Research Council \\ Laboratory of Molecular Biology, Cambridge CB2 2QH, England
}

\begin{abstract}
Mutations in the gene unc-53 of Caenorhabditis elegans result in behavioral and anatomical abnormalities. Immunocytochemistry and electron microscopy revealed neuroanatomical defects in all main longitudinal nervous tracts. Whole tracts were found to be misguided in specific ways suggesting that unc-53 affects pioneering axons. The four lateral microtubule cells (LMs), which are probably pioneering neurons, were examined in greatest detail. In the mutants, the processes of the LMs leave their normal position on the body wall and terminate prematurely. Examination of five unc-53 alleles for penetrance and expressivity of these defects revealed a spatial restriction in the requirement for unc-53.

The morphology and positioning of the branch of the posterior lateral microtubule cells (PLMs) were also examined. In wild-type animals, the PLM branches lack the ultrastructural specializations of the main process, which include large microtubules, apposition to the cuticle, and a polarized extracellular matrix (the mantle). Two differences were noted in unc-53 mutants. First, a majority of PLMs branch at random and display an abnormally enlarged branching point and branch cross section. The unusual branch morphologies correlate with branch position, rather than PLM length. Second, the ectopic branches display the specific ultrastructural features characteristic of the main process. Furthermore, after entering the ventral nerve cord, the abnormal branches constantly change position relative to the other processes and the hypodermis, retaining their specialized microtubules throughout, but displaying a mantle only when in direct contact with hypodermis.

Taken together, these observations suggest that the differentiated features of the PLMs, including process length, branch position, intracellular branch morphology, and surrounding extracellular matrix, are locally specified by cellextrinsic cues, some of which require unc-53.
\end{abstract}

\footnotetext{
Received Aug. 18, 1992; revised Apr. 1, 1993; accepted Apr. 7, 1993.

This work was supported by the Canadian National Science and Engineering Research Council (NSERC Operating Grant OGP 0121351), the Swiss National Science Fund (Requete 823A-25064), the Medical Research Council's Laboratory of Molecular Biology in Cambridge, and McGill I Iniversity. We thank Marty Chalfie, Ron Chase, Bob Levine, Jonathan Ewbank, and in particular Tom Barnes for critically reading the manuscript. We also thank Mark Bretscher and Jonathan Hodgkin, John White and Steven Hird for comments on a previous version of the manuscript. We thank Bob Horvitz and Thierry Bogaert for unc-53 alleles.

Correspondence should be addressed to Siegfried Hekimi, Department of Biology, McGill University, 1205 Docteur Penfield Avenue, Montréal, PQ, Canada $\mathrm{H} 3 \mathrm{~A} 1 \mathrm{~B} 1$.
}

Copyright (c) 1993 Society for Neuroscience 0270-6474/93/134254-18\$05.00/0
[Key words: neuronal differentiation, axonal pathfinding, collateral branch induction, cytoskeleton, extracellular matrix, touch receptor neurons, nematode]

The structure of the adult nervous system of the nematode Caenorhabditis elegans is highly invariant and has been described at the single-cell level (White et al., 1986). In addition, this organism can tolerate mutations that lead to extremes of behavioral impairment while conserving viability and fertility (Brenner, 1974). These characteristics make it possible to identify and study behavioral mutants with severe underlying developmental defects (Hedgecock et al., 1990; McIntire et al., 1992). Detailed examination of such defects can then lead not only to the formulation of hypotheses as to the wild-type function of the gene, but also to the elucidation of normal developmental mechanisms through the analysis of the pattern of secondary neuroanatomical defects introduced by the primary genetic lesion. For example, Hall and Hedgecock (1991) have brought together observations of mutants of the axonal guidance gene unc-5 (Hedgecock et al., 1990) with observations of mutants of unc-104, a gene required for synaptic vesicle transport, to suggest the involvement of a diffusible factor in the guidance mechanisms required for neuromuscular junction formation.

The six touch receptor neurons of $C$. elegans, also called microtubule cells (MCs), are a group of mechanosensory cells with common morphological, functional, and molecular features (Chalfie and Sulston, 1981; Way and Chalfie, 1988; Savage et al., 1989). They have been extensively used as a model to study the genetics of neuronal differentiation. An important group of genes has been shown to be specifically required for their differentiation (Chalfie and $\mathrm{Au}, 1989$ ). Those genes that have been studied in detail appear to act in a cell-intrinsic fashion (Savage et al., 1989; Way and Chalfie, 1989; Finney and Ruvkun, 1990; Driscoll and Chalfie, 1991; Hamelin et al., 1992). This could suggest that cell autonomy of differentiation is the rule for these cells or even for all neurons in $C$. elegans. However, mutants with locomotory defects cannot easily be tested for mechanosensitivity and therefore a screen for touch-insensitive mutants necessarily discriminates against mutations affecting a larger group of neurons. Furthermore, in the case of the postembryonic anterior ventral microtubule cell (AVM), experiments based on laser microsurgery have clearly demonstrated the existence of cellular interactions during axonal pathfinding (Walthall and Chalfie, 1988) and collateral branch induction (Chalfie et al., 1983).

Mutations in the gene $u n c-53$ result in locomotory incoordination and defective egg laying (Brenner, 1974; Trent et al., 
1983). Reported anatomical defects include defects in the excretory canals (Hedgecock et al., 1987) and the male bursa (Hodgkin, 1983). In addition, observations of posterior lateral microtubule cells (PLMs) of abnormal morphology have been reported (Siddiqui, 1990). Given the widespread nature of the defects, it is not clear whether the normal development of the affected structures specifically requires $u n c-53$ or if these structures are more indirectly affected by an unknown primary pathological condition of these mutants.

Here, observations of the neuroanatomy of a series of alleles of $u n c-53$ are reported. They suggest a specific role for $u n c-53$ in nervous system development and indicate that several aspects of the differentiation of the PLMs and other MCs are controlled by cell-extrinsic determinants.

\section{Materials and Methods}

Strains and genetics. Nematodes were maintained as described by Brenner (1974). The mutations lon-1(e185), dpy-3(e27), and unc-53(e404) were described by Brenner (1974). Three alleles of unc-53 (n166, n152, and $n 569$ ) were described by Trent et al. (1983). unc-53(e2432) was isolated by Thierry Bogaert (personal communication). Double mutants were constructed by mating lon-1(e185)/ + or $d p y-3(e 27) /+$ males to $u n c-53(n 166)$ hermaphrodites and picking from the $\mathrm{F} 2$ generation long and uncoordinated or dumpy and uncoordinated animals, respectively.

Immunocytochemistry. The antiserum (anti- $\beta$ ) used in the present study, and fixation and staining methods have been described (Hekimi, 1990).

Briefly, worms were collected from $9 \mathrm{~cm}$ Petri dishes by washing with M9 buffer or distilled water. The following procedure was then performed in $1.5 \mathrm{ml}$ Eppendorf tubes. Worms were fixed for $24 \mathrm{hr}$ at $4^{\circ} \mathrm{C}$ with $4 \%$ paraformaldehyde in $0.2 \mathrm{M}$ phosphate buffer $(\mathrm{pH} 7.4)$. They were then washed in PBS, partially disrupted with a glass homogenizer, and incubated for $1-3 \mathrm{hr}$ in $1 \mathrm{ml}$ of $500 \mathrm{U} / \mathrm{ml}$ collagenase (Sigma, type IV) at $37^{\circ} \mathrm{C}$ with gentle resuspension of the worms every $30 \mathrm{~min}$. Anti- $\beta$ was used at a dilution of $1: 100$ with $1 \%$ nonfat, dehydrated milk and $1 \%$ Tween 20 in PBS. The secondary antibody, FITC-conjugated affinity-purified goat anti-rabbit serum, from Sigma, was used at a dilution of $1: 100$ with $1 \%$ milk and $1 \%$ Tween 20 in PBS. Best results were obtained with incubation of at least $24 \mathrm{hr}$ with both antisera.

Electron microscopy. The procedures for animal preparation, fixation, and sectioning were performed essentially as described by White et al. (1986). Briefly, in order to make them assume a straight posture, the worms were chilled on ice in a small volume of $0.1 \mathrm{M}$ HEPES buffer. They were then fixed in $1 \% \mathrm{OsO}_{4}$ for $5 \mathrm{~min}$ on ice followed by $1 \mathrm{hr}$ at room temperature. After fixation, the worms were put on a thin layer of $1 \%$ agar and cut in half, either just anterior or just posterior to the vulva. The cut worms were covered with a drop of molten $1 \%$ agar and blocks of agar containing up to three aligned worms were cut out. These were dehydrated through the following series of solutions of increasing acetone concentration: $15 \%$ for $5-10 \mathrm{~min}, 30 \%$ for $5-10 \mathrm{~min}, 60 \%$ for $5-10 \mathrm{~min}, 90 \%$ for $10-15 \mathrm{~min}, 95 \%$ for $10-15 \mathrm{~min}, 100 \%$ for up to 3 $\mathrm{hr}$ with three washes. The following treatments were then all performed at $60^{\circ} \mathrm{C}$. The blocks were transferred to a $2: 1$ acetone:araldite (CIBA) solution for $30 \mathrm{~min}$, followed by a 1:2 acetone:araldite solution for 30 min and then $2 \times 30 \mathrm{~min}$ in $100 \%$ araldite. Finally, they were put into fresh $100 \%$ araldite and left to polymerize overnight in gelatin capsules. An LKB ultratome III was used with a diamond knife to cut transverse serial sections of approximately $50 \mathrm{~nm}$ thickness. The sections were collected on Formvar-coated G50 copper/palladium grids. The grids were then poststained with $5 \%$ uranyl acetate for $10 \mathrm{~min}$ at $60^{\circ} \mathrm{C}$, washed with double-distilled water, and air dried. Finally they were stained with lead citrate stain (Reynolds) for $2 \mathrm{~min}$, washed, and air dried.

Scoring of neuroanatomical features. The length of neuronal processes was not directly measured; instead we determined an extension index in the following way. In both the wild type and the mutants, the cell bodies of the PLMs are located in tail ganglia, posterior to the anus, and their anterior processes terminate slightly anterior to the vulva. Only those animals in which both the PLM cell body and the vulva were stained and visible were used for scoring. The absolute length of the PLMs was not directly measured, but instead for each PLM a subjective judgement was made as to whether its anterior process extended beyond the midpoint of the distance between its cell body and the vulva.
The PLM extension index in a given genotype is thus the proportion of the observed PLMs that succeed in extending beyond the midpoint. The extension index of the anterior lateral microtubule cells (ALMs) was scored in a similar way between the back of the terminal bulb of the pharynx where the pharyngeal-intestinal valve is situated and the tip of the pharyngeal procorpus.

Body length measurements were performed in the following way. Animals were fixed with paraformaldehyde as described above, which induced a generalized autofluorescence in the fixed tissues. Photomicrographs based on this fluorescence were made, and the length of the animals measured with a ruler on these micrographs. These measurements are considered accurate to $\pm 5 \%$. The length of the tail spike was not included in the measurements. The posterior body length was taken as the length between the vulva and the base of the tail spike. To obtain I 1 larvae, eggs were obtained from gravid adults by hypochlorite treatment, hatched in M9 buffer overnight, and fixed the next day.

\section{Results}

Defects in the major process tracts

The anatomy of the nervous system of five unc-53 mutants (e2432, n569, n166, n152, and e404) was examined using immunocytochemical staining with an antiserum with which all neurons of $C$. elegans can be stained, including axons and cell bodies (Hekimi, 1990). The general morphology of process bundles can be easily assessed with this method. Single neurons within bundles, however, cannot be resolved in most cases. In all five alleles we have examined, we have observed abnormal ventral, dorsal, and sublateral nerve cords, as well as abnormal MCs (Table 1). In addition, a variety of abnormal neuroanatomical features involving unidentified neurons were also seen (see below).

Dorsal cord. In the wild type, the dorsal nerve cord runs as a coherent bundle on the left side of a dorsal hypodermal ridge, which separates the two dorsal muscle quadrants. In unc-53 mutants, a small process bundle is frequently seen to run on the right side of the ridge as well (Fig. 1 a). In the head of the wild type, the dorsal cord runs on the right rather than on the left of the hypodermal ridge, while in the head of unc-53 mutants, processes can also be found on the left side (Fig. 1b). The ectopic processes are sometimes continuous along the entire length of the dorsal hypodermal ridge but in other instances they extend only over a short distance. In the latter cases the processes appear to originate from the dorsal cord and refasciculate with it at another point, or stop. In no case were the exact identities of ectopic processes established.

Ventral cord. In the wild type the ventral nerve cord is composed of two process bundles running on either side of a large ventral hypodermal ridge. The right bundle is the largest and also comprises the cell bodies of motor- and interneurons. In unc-53 mutants, the left bundle is frequently strongly abnormal. Phenotypes include complete absence (Fig. 1a), abrupt premature termination (Fig. $1 c$ ), and fasciculation with the right side of the cord (Fig. 1d).

Sublateral cords. In the anterior part of the animal, the dorsal and ventral sublateral cords run in the middle of the dorsal and ventral muscle quadrants, respectively. They are therefore placed on each side of the dorsal and ventral midlines. In the region just anterior to the vulva they turn toward the lateral hypodermis. In unc-53 mutants, two types of abnormality of the sublateral cords can be observed. Cords are sometimes entirely missing. Figure $1 b$ illustrates the case of the absence of a right dorsal sublateral cord. In addition, we observed sublateral cords prematurely turning laterally. In this case they frequently turn to the wrong side, ventrally or dorsally instead of laterally, and 

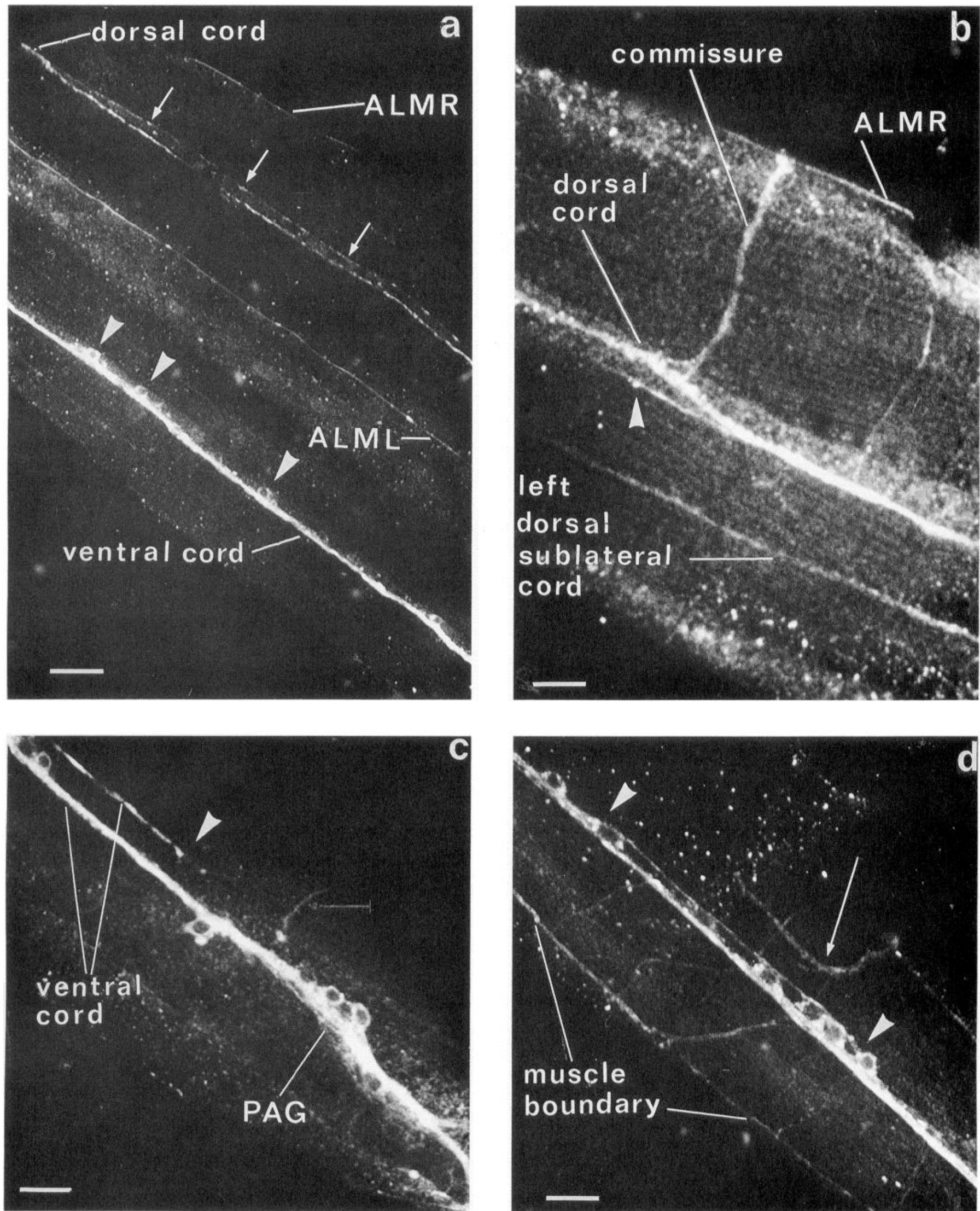

Figure 1. Neuroanatomical abnormalities in the dorsal and ventral cords of unc-53(e404) mutants. The animals were stained with an antiserum that stains the entire nervous system (Hekimi, 1990) and were visualized by laser scanning fluorescence confocal microscopy. The anterior of the animal is on the upper left in all four pictures and the scale bars are $10 \mu \mathrm{m}$, except in $c$, where it is $5 \mu \mathrm{m}$. $a$, In this preparation the animal's body wall has been ruptured along one of the ventral muscle boundaries and the body wall lies unfolded with the internal side against the microscope 
Table 1. Frequency of neuroanatomical defects in unc-53 mutants and in the wild type

\begin{tabular}{|c|c|c|c|c|c|c|}
\hline & \multirow[b]{2}{*}{ Wild type } & \multicolumn{5}{|l|}{ unc -53} \\
\hline & & $e 2432$ & $n 569$ & $n 166$ & $n 152$ & $e 404$ \\
\hline Dorsal cord & $0 / 85$ & $50 / 105$ & $15 / 69$ & $22 / 66$ & $9 / 27$ & $10 / 72$ \\
\hline Ventral cord & $0 / 73$ & $67 / 79$ & $23 / 45$ & $37 / 60$ & $19 / 29$ & $41 / 66$ \\
\hline \multicolumn{7}{|l|}{ Dorsal } \\
\hline sublateral cord & $3 / 50$ & $16 / 59$ & $9 / 13$ & $6 / 20$ & $2 / 14$ & $10 / 50$ \\
\hline \multicolumn{7}{|l|}{ Ventral } \\
\hline sublateral cord & $1 / 54$ & $1 / 47$ & $5 / 9$ & $0 / 15$ & $0 / 14$ & $0 / 28$ \\
\hline ALM & $0 / 259$ & $171 / 182$ & $100 / 164$ & $49 / 49$ & $88 / 89$ & $174 / 174$ \\
\hline PLM & $0 / 170$ & $465 / 467$ & $181 / 185$ & $131 / 132$ & $133 / 136$ & $107 / 107$ \\
\hline AVM & $1 / 92$ & $53 / 53$ & $41 / 42$ & $8 / 8$ & $13 / 13$ & $9 / 9$ \\
\hline PVM & $1 / 22$ & $44 / 44$ & $22 / 24$ & $7 / 7$ & $1 / 1$ & $1 / 1$ \\
\hline
\end{tabular}

The nervous system of five unc. 53 mutants and the wild type was visualized by immunocytochemistry. The structures of eight distinct parts of the nervous system were scored as normal or abnormal. The table lists the frequencies of abnormal structures in various alleles and the wild type. The degree and nature of the morphological abnormalities observed were variable for each structure (see text for description). The three dorsal and the one ventral sublateral cords that were scored as abnormal in the wild type appeared to be entirely missing. The single AVM and PVM that were scored as abnormal in the wild type were slightly short. The antiserum used for immunocytochemistry stains the entire nervous system (Hekimi, 1990). The AVM and PVM neurons run within large process bundles. For this reason their processes could frequently not be visualized unambiguously. These neurons were scored as abnormal only when their processes could be identified and appeared too short. In the three alleles, n166,n152, and $e 404$, in which the degree of shortening of ALM and PLM is particularly severe (see text and Fig. 6), AVM and PVM could only very rarely be visualized. It is possible that this was due to their processes being extremely short.

fasciculate with the ventral or dorsal cord (Fig. 2a). The complete absence of ventral or dorsal sublateral cords was also observed in the wild type at a very low frequency (Table 1).

Abnormalities involving unidentified neurons. The antiserum used in this study stains a group of varicosities in the middle of the four muscle quadrants in the head very intensely. Figure $2 b$ illustrates the wild-type condition. The identity of the neurons stained in this way is unknown but is likely to correspond to one of the classes of sensory cells found in this location (White et al., 1986). In unc- 53 mutants the series of stained varicosities are frequently shortened and/or do not remain in the middle of the quadrant but turn laterally (Fig. $2 c$ ).

Unidentified neuronal processes can also be observed to run in various ectopic locations. Observed instances include the dorsal and ventral muscle boundaries (Fig. 1d), the middle of the posterior ventral muscle quadrants (Fig. 1d), and along existing nerve bundles, such as the bundle associated with the canal cell process (not shown).

\section{Length and placement of the MCs' processes}

Wild-type morphology. The morphology of the MCs has been described in detail (Chalfie and Sulston, 1981). However, to facilitate the description of their anatomy in the mutants a schematic of the wild-type morphology is shown in Figure 3 and described in the caption.

Length and placement of main processes. In the five unc-53 mutants examined, the anterior process of the PLMs and ALMs are shortened (Fig. 4). While in the most severe allele (e404) the PLM processes may be only a few tens of micrometers long, in weaker alleles (e.g., $n 569)$ some individuals possess PLMs of almost wild-type length ( $>350 \mu \mathrm{m}$ ) (see below for quantitative comparisons between alleles). In contrast to the situation for the PLMs, the shortening of the ALMs is restricted to the last third of their outgrowth. In all alleles the ALMs extend at least to the back of the pharynx, at the level of the pharyngeal-intestinal valve. In the strongest alleles they frequently do not

\section{$\leftarrow$}

slide. The inner organs, including the gut and gonads, are lost in this type of preparation. The body wall includes the hypodermis, muscles, and nervous system, ensheathed between the external cuticle and an internal basal lamina. In this mutant animal, the ventral and dorsal cords are abnormal. The mutant dorsal cord displays ectopic processes running along the right side of the hypodermal ridge (indicated by three arrows). In the wild type (not shown), the dorsal nerve cord runs exclusively along the left side of a dorsal hypodermal ridge. The cellular origin of these processes is not known. The ventral cord of the mutant shown in $a$ displays only the right nerve bundle. The wild-type ventral nerve cord is composed of two process bundles: a major bundle on the right and a minor bundle on the left of a central hypodermal ridge. The normal aspect of these bundles and their relative thickness can be seen in $c$. The right side also contains neuronal cell bodies located between the bundles. These cell bodies are present in the mutant and identified in the figure by arrowheads. In the figure, the major process bundle appears on the left of the cell bodies because of the way the preparation is visualized (see above). $b$, In the head of the wild type (not shown), the dorsal cord changes position and runs on the right rather than on the left of the central hypodermal ridge. This change is also observed in unc- 53 mutants as shown in $b$. However, ectopic processes are also frequently found on the left of the ridge (arrowhead). Ectopic processes on either side of the ridge either refasciculate with the cord or stop abruptly. The latter case is illustrated here (arrowhead). The mutant animal in the figure is also missing its right sublateral cord. The sublateral nerve cords are normally found in the middle of the muscle quadrants on both sides of the ventral and dorsal nerve cords. In unc-53 mutants individual dorsal or ventral sublateral cords are frequently entirely missing or misguided (see also Fig. 2). In the figure, the left sublateral cord can be clearly seen while the right cord appears to be entirely missing. The animal is prepared in whole-mount and lies on its ventral side. $c$, Abnormal stop (arrowhead) of the left bundle (appearing here on the right) shortly before the first ganglion of the tail, the preanal ganglion $(P A G)$. The animal is prepared in whole-mount and lies on its dorsal side. $d$, In this mutant animal the left bundle of the ventral cord fasciculates with the right side (arrowheads) both anteriorly and posteriorly of a region where it runs at its correct location. In the wild type, the right and left bundles run completely independently. $d$ also shows unidentified processes running at ectopic locations such as the muscle boundary and within the left muscle quadrant (arrow). The animal is prepared in whole-mount and lies on its dorsal side. $A L M R$, anterior lateral microtubule cell right; $A L M L$, anterior lateral microtubule cell left. 


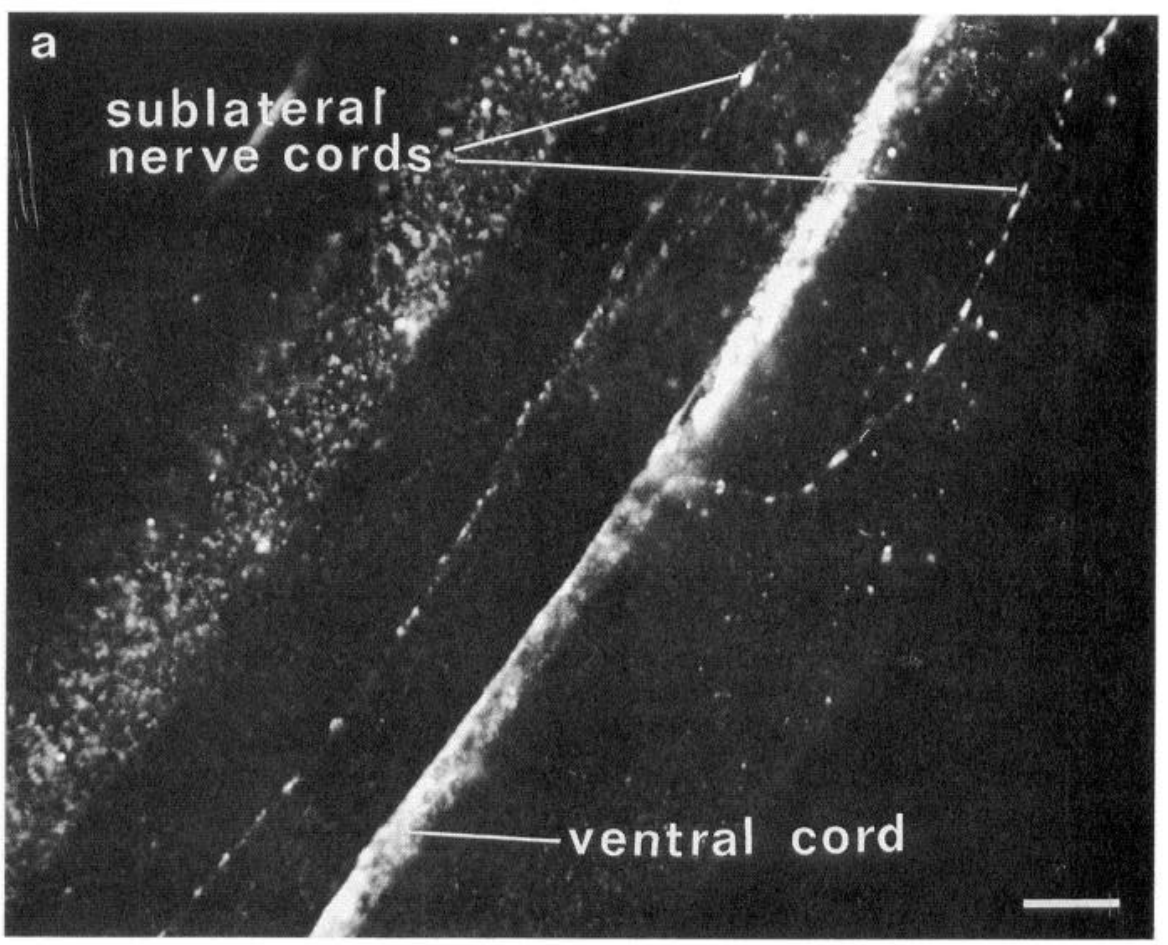

Figure 2. Abnormal turn by a ventral sublateral nerve cord in an unc-53(n569) mutant $(a)$, and abnormal turns of sensory processes in the head of an unc53(n569) mutant (c) compared to wild type $(b)$. In the three panels of this figure the anterior of the animal is toward the top. The animals were prepared in whole-mount. In $a$ the animal lies on its dorsal side; in $b$ and $c$, on one of its lateral sides. $a$, In the wild type, the ventral and dorsal sublateral nerve cords start in the head of the animal and run in the middle of the ventral and dorsal muscle quadrants, respectively. In the middle of the animal, near the vulva, they turn laterally. One of the defects observed in the dorsal and ventral sublateral cords of $u n c-53$ mutants are turns that are both premature and to the wrong side. The picture illustrates a mutant phenotype where the left ventral sublateral cord turns ventrally instead of laterally and fasciculates with the ventral cord. The right bundle is unaffected. The left side of the ventral cord is missing in this animal. $b$, The antiserum used in this study stains a group of varicosities in the middle of the four muscle quadrants in the head very intensely (Hekimi, 1990). The arrow points to one of them. The anteriormost tip of the animal is indicated by an arrowhead at the top of the picture. A patch of light emanating from the intensely stained nerve ring (n.r.) can be seen. $c$, In $u n c-53$ mutants the series of varicosities is often extremely shortened and/or turns toward the lateral hypodermis (lat. hyp.). The arrow points to one of the ventral series. The dorsal series can be seen to turn slightly before. Scale bars: $a, 10 \mu \mathrm{m} ; b$ and $c, 30 \mu \mathrm{m}$.
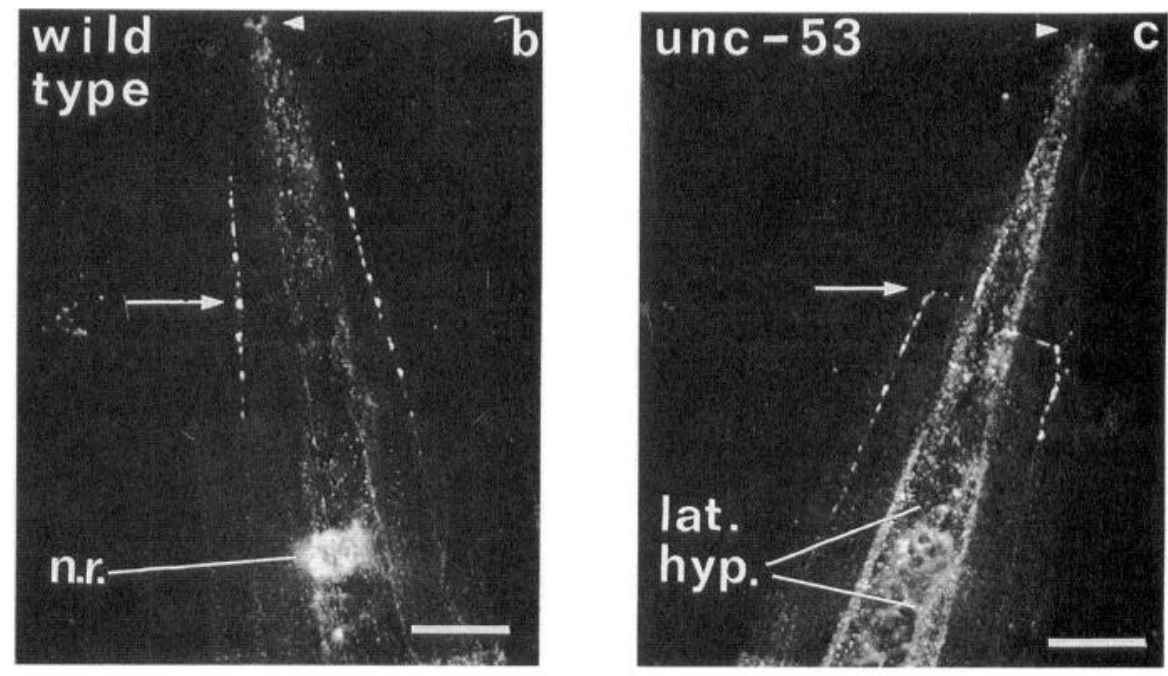

extend much beyond this point, while in the weakest alleles they may extend beyond the anterior bulb of the pharynx.

In addition to their shortening, the processes of both pairs of cells frequently become misguided before stopping (Fig. 5). While in the wild type the processes of the lateral microtubule cells (LMs) always appear straight, they are often meandering in the mutants (Fig. $5 a$ ), with a general tendency to drift ventrally (Fig. $5 b$ ). In the most extreme cases they drift away until they fasciculate with a large process bundle, the ventral cord in the case of the PLMs and the nerve ring in the case of the ALMs (Fig. $5 c$ ). In the strongest alleles some PLM processes get deflected or misguided immediately after outgrowth, such that they don't leave the ganglion anteriorly but proceed directly into the ventral cord through the major commissure in the tail. In contrast again, the ALM processes always remain in position for most of their length and will only start meandering when they reach the level of the pharyngeal-intestinal valve.

The two ventral MCs arising postembryonically, AVM and PVM (posterior ventral microtubule), run within the ventral nerve cord for most of their length. This makes it difficult to visualize them with the antiserum used in this study, which stains all processes. However, all MCs are more intensely stained than other cells with our method. This made it sometimes possible to visualize them even within process bundles, particularly in animals with overall weak staining. When they could be unambiguously scored, both AVM and PVM appeared shortened in all alleles (Table 1). AVMs were similar to the ALMs in that they never stopped before the beginning of the head region, corresponding to the level of the retrovesicular ganglion (RVG) in the ventral cord, and frequently continued anteriorly 


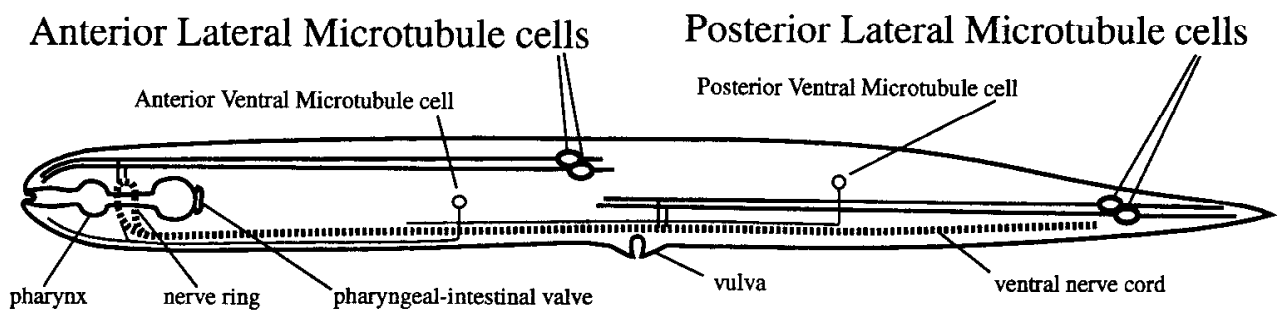

Zone of unc-53

requirement for

ALMs and AVM
Zone of unc-53 requirement for PLMs

Zone of unc-53 requirement for PVM

\section{$0.1 \mathrm{~mm}$}

Figure 3. Schematic of the placement and morphology of the six MCs in the wild type, and regions of the body in which the MCs require wildtype unc-53 activity for normal anatomy. The lateral MCs are born embryonically and the ventral MCs are born postembryonically. The cell bodies of the two ALMs are positioned bilaterally on the dorsal body wall, in isolation from other cell bodies. The cell body of the AVM is also isolated on the right side of the body wall. The cell bodies of the PLMs are found in bilaterally symmetrical ganglia behind the anus, and the cell body of the PVM is found in the left posterior lateral ganglion, on the side of the body wall. The two ventral MCs (AVM and PVM) run in the ventral cord for most of their length (for clarity they are shown respectively below and above the cord in the figure). The ALMs have only a very short or entirely missing posterior process. Their anterior process branches into the nerve ring and peters out at the level of the anterior pharyngeal procorpus. AVM has a single process that branches into the nerve ring and also peters out at the level of the procorpus. In the figure the processes appear curved in the narrowing head, but their relative circumferential position on the body wall of the animal remains unchanged. The PLMs have a posterior process that extends into the tail spike. The anterior process branches into the ventral cord in the region of the vulva, generally slightly posterior to the vulva proper, and extends to the level of the ALM cell bodies. PVM has a single process, does not branch, and terminates anteriorly of the AVM cell body. The anterior and posterior MCs appear to have different requirement for unc-53 activity (see also text). In all unc-53 mutant animals observed the ALMs and AVM extend at least to the level of the pharyngeal-intestinal valve. In the region of the pharynx they will stop at various distances before their normal end point depending on the severity of the allele. In contrast, in the most severe alleles, the PLMs and PVM display extreme shortening with sometimes an apparently complete absence of outgrowth. In weaker alleles, they grow to various lengths, depending on the severity of the allele. The dependence on unc-53 of the posterior process of the PLMs is unclear and is therefore indicated with a broken line.

for various distances. In contrast, PVM was practically ncver observed to extend beyond the vulva, and sometimes could be seen to stop immediately after entering the ventral cord.

A possible, though unlikely, explanation for the finding of the shortening of the MCs is that the processes are not actually shortened but have lost the ability to be stained by anti- $\beta$. In order to eliminate this possibility, a few mutants $(3 \times e 404$ mutants and $3 \times e 2432$ mutants) were also examined by electron microscopy (EM). This revealed a pattern of shortening and misplacement identical to that observed by immunocytochemistry (data not shown). The landmarks used to assess anteroposterior level in these EM reconstructions included the vulva and the intestinal muscle.

Among the MCs, only the PLMs have a prominent posterior process. Along with other processes it runs into the very narrow tail spike, a structure that almost invariably sustains serious damage during the staining procedure. We were therefore not able to make unambiguous observations about possible shortening or misplacement of the posterior process. However, extremely shortened posterior processes, which would have been observed, were not seen.

Allelic series. The anterior processes of the ALMs or the PLMs were abnormally shortened in almost all cases in which their length could be scored. In the other observed cases, they had fasciculated with the ventral cord or the nerve ring, which prevented scoring of their length. Table 1 shows that, taken together, the penetrance of the two types of defects affecting the $\mathrm{MCs}-$ shortening and misguidance-is almost complete for all alleles. To obtain a measure of the relative severity of the alleles, the expressivity of three distinct defects - the reduced length of the ALMs, the reduced length of the PLMs, and the abnormal placement of the PLMs-was scored in all alleles and in the wild type (Fig. 6). The measure of expressivity chosen for the length defects was the enumeration for each allele of the fraction of the ALMs or PLMs that succeed in extending their processes beyond the vulva (see Materials and Methods and Fig. 6 caption). We call this measure an extension index. As a measure of guidance, we scored the frequency with which misplacement resulted in abnormal fasciculation of the PLMs with the ventral nerve cord. This all-or-none phenomenon represents only an extreme in a continuum of misplacement. However, it can easily be scored and its frequency is taken here as a measure of the degree of misguidance. We call this measure a guidance index.

A possible weakness of our way to determine expressivity is that the extension index makes no distinction between axons that just exceed the half-way distance to the vulva and those that would have reached the wild-type length. This could be a problem if, in a given allele, the distributions of the actual lengths of the PLM and ALM axons were showing two or a small number of sharply separated modal values. It is likely that we would have noticed such a distribution. In fact, we observed a relative broad distribution of axonal lengths around a mean value typical for the allele.

The much more abrupt transition in the middle of the series of scores of ALM length may possibly be attributable to an observational bias due to the intensely stained nerve ring, which interfered with the observation of ALMs of intermediate lengths. Because ALMs whose termination point could not be unambiguously observed were not scored at all, it is possible that in weak alleles a relative excess of long ALMs, and in strong alleles a relative excess of short ALMs, has been scored.

Figure 6 shows that similar allelic series are obtained for qualitatively very different phenotypes. Taken together, the fact that all alleles display the same set of abnormalities, the fact 
Figure 4. Abnormally short PLM and ALM in unc- 53 mutants. $a$, PLM in an unc-53(e2432) mutant. The anterior process is only about $100 \mu \mathrm{m}$ long, more than three times shorter than in the wild type. The "PLM" label points to the position of the cell body that is out of the plane of focus. The arrowhead points to a branch with an abnormal morphology (see text), which continues out of the plane of focus to join the ventral nerve cord. $b$, Short ALM in an unc53 (n166) mutant, stopping immediately after the nerve ring. The " $A L M$ " label points to a varicosity at which a branch into the nerve ring is made. The position and length of the ALM process can be related to the nervous structures visible in the micrograph. The punctated staining labeled "sensory" corresponds to unidentified but presumably sensory processes running in the middle of the muscle quadrants (see text and Fig. 2). In the wild type, the ALMs extend beyond the anteriormost end of these processes. The amphid commissure, a bundle of processes that leaves the ventral cord shortly posterior to the nerve ring, can be seen. The nerve ring is in a different plane of focus, but can be seen as a region of diffuse light anterior to the amphid commissure. The intensely stained structures visible in the lower part of the image and indicated by a question mark do not correspond to known structures and are sporadically found in all genotypes. ant., anterior; post., posterior; ventral sublat., ventral sublateral nerve cord. Scale bars, $20 \mu \mathrm{m}$.
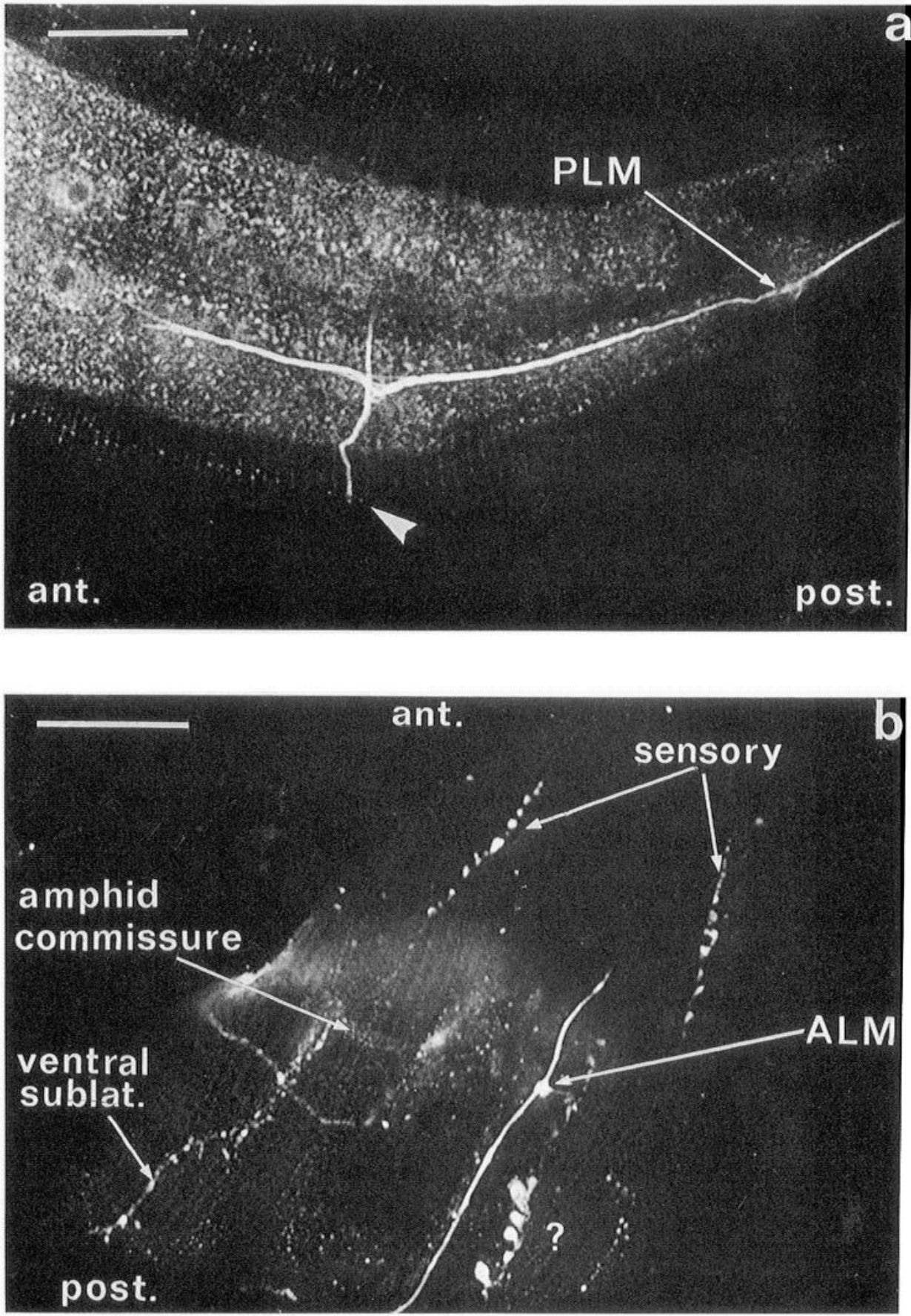

that these alleles can be easily ordered based on their severity, as well as the consistency between the series, suggest strongly that the ranking of alleles shown in Figure 6 represents a similar ranking of residual gene activities.

These series do not directly reveal the nature of the null phenotype of the gene. All the available mutations, however, have qualitatively similar effects. Given that mutations in unc-53 arise with relatively high frequency (Trent et al., 1983), it is likely that the observed effects correspond to reduction in gene activity rather than to the gain of a function unrelated to the wild-type activity. Our observations suggest, therefore, that there is a direct quantitative relationship between the amount of unc53 gene activity and the extension and placement of the LMs' processes.
PLMs in mutants with altered body length. The ALMs and PLMs show a very different requirement for unc-53 activity, the ALMs requiring it only for the last part of their outgrowth. The ALMs and PLMs, however, are very similar cells and they differ mostly by their different placement in the body and their connections to different sets of neurons. This suggests that the differences in their requirement for $u n c-53$ activity reflect an involvement of $u n c-53$ in spatially localized interactions. Another possibility is that there is a quantitative difference in the requirement of the two cell types for unc- 53 activity. It is possible that $u n c-53$ mutations affect the general condition (health) of the MCs, impairing their ability to synthesize the structural components of their processes and that, for some reason, the ALMs require less activity for their biosynthetic processes than 
a
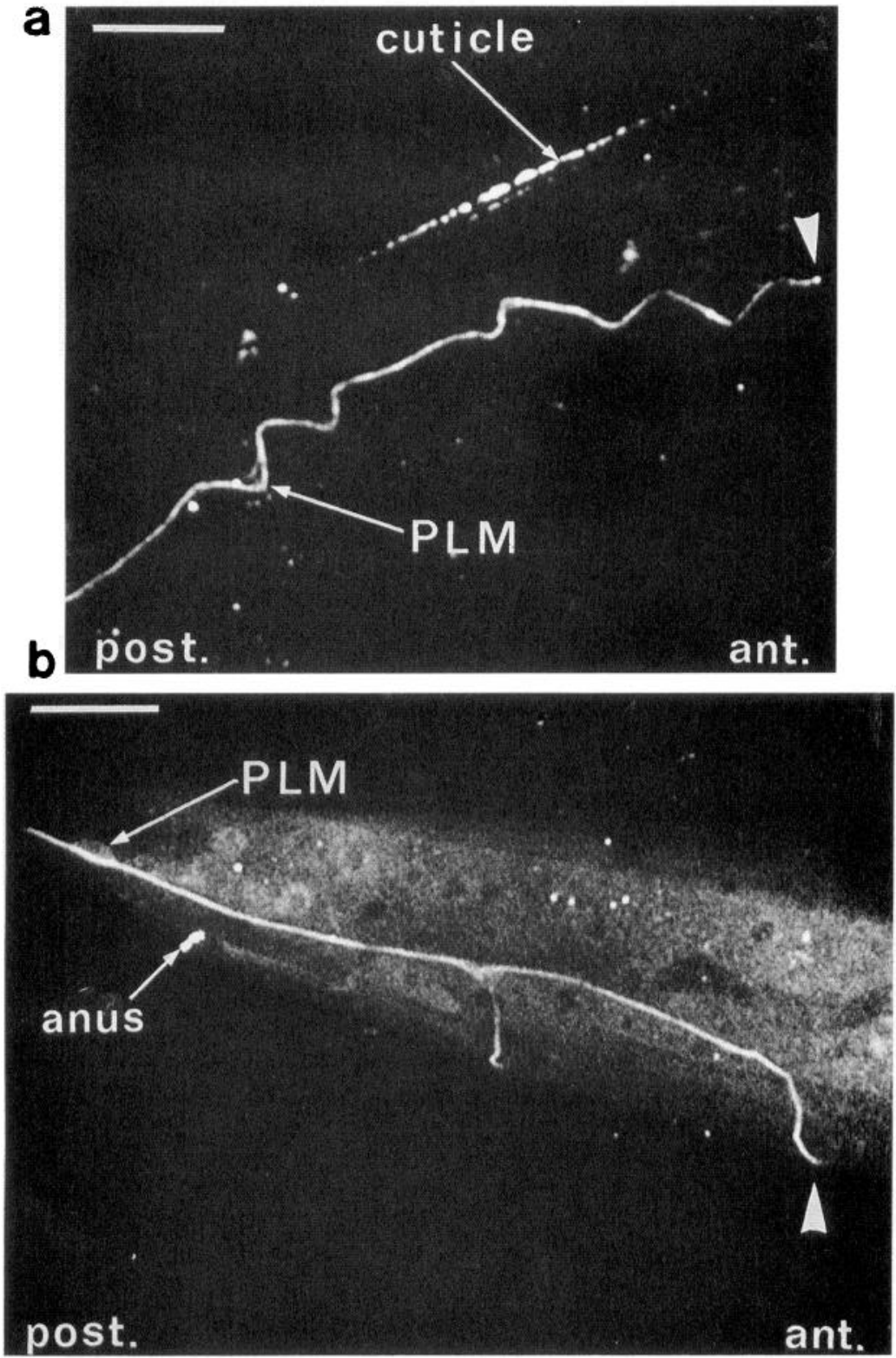

C

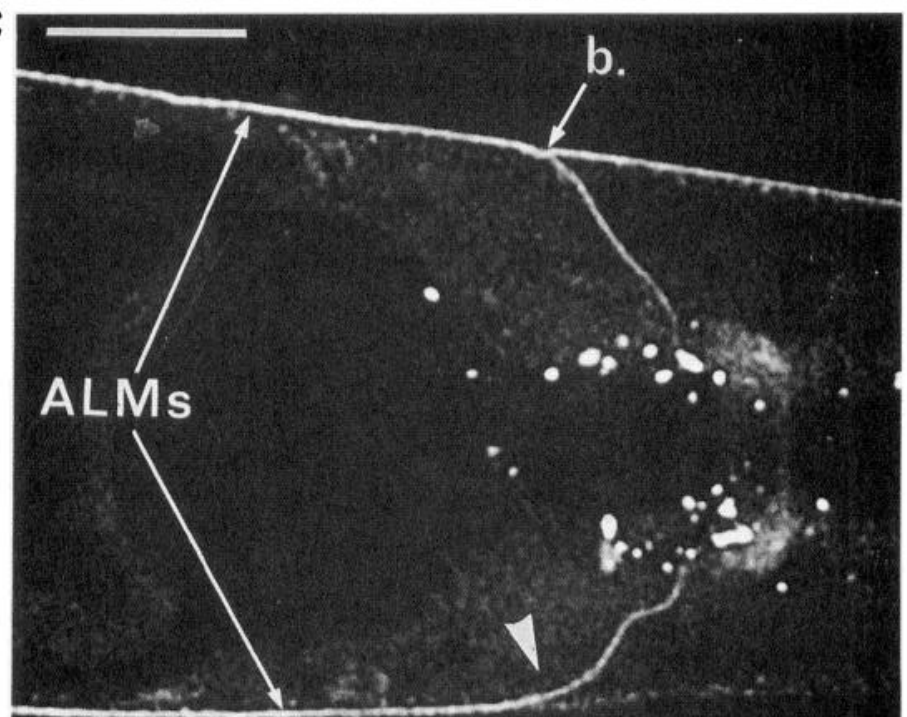

post. ant.
Figure 5. Defective guidance of PLMs and ALMs in unc-53 mutants. $a$, A PLM in an $n 166$ mutant. The anterior process starts meandering immediately after leaving the cell body (arrow labeled " $P L M$ ') and stops abruptly (arrowhead). The arrow labeled "cuticle" points to nonspecific deposits associated with cuticular ridges on the surface of the animal that show that the meandering aspect of the PLM is not due to deformation of the hypodermis sustained during preparation. $b$, Anterior process of a PLM in an n166 mutant drifting ventrally and joining the ventral cord (arrowhead), which itself is not seen in this plane of focus. $c$, The main process of an ALM in an $n 152$ mutant turning into the nerve ring (arrowhead). The weak staining anterior to the turn is on the external cuticle and is unrelated to the ALM. The other ALM (top of the image) makes a normal branch (arrow labeled " $b$ ") at the same level. The arrows labeled " $A L M$ " " point to the main processes. ant., anterior; post., posterior; $b$., ALM branch. Scale bars, $20 \mu \mathrm{m}$. 

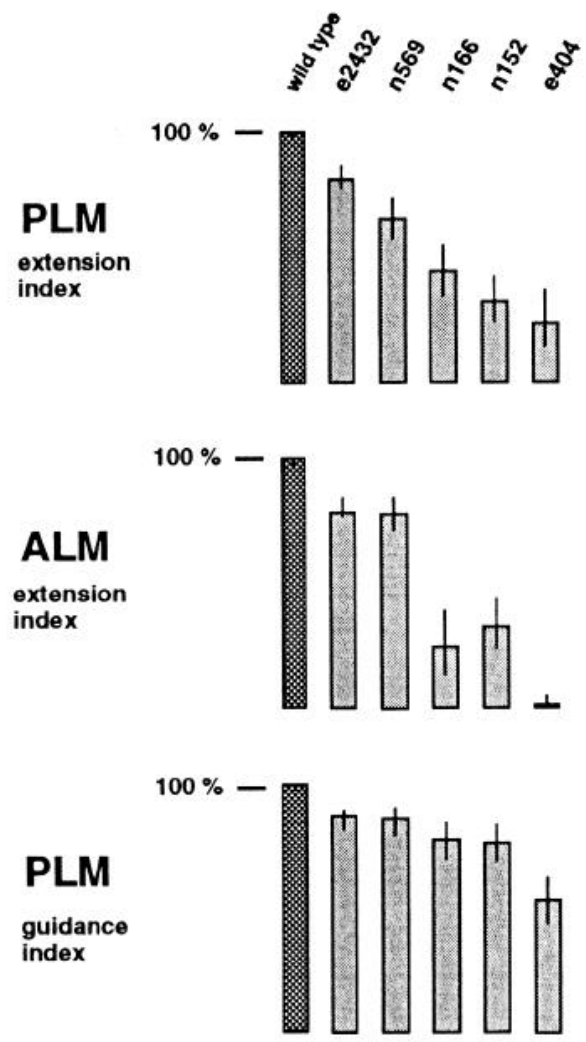

Figure 6. Expressivity of three different mutant phenotypes in five unc-53 alleles and in the wild type. The PLM extension index is established by scoring the fraction of the observed PLMs that succeed in extending beyond the midpoint between the PLM cell body and the vulva, which corresponds to approximately half their normal anterior extension. The ALM extension index is established by scoring the fraction of the ALMs that extend beyond the nerve ring, corresponding to the midpoint of the region over which their extension is affected in the strongest alleles. The PLM guidance index is established by scoring the fraction of the PLMs that do not fasciculate with the ventral cord. The error bars represent the $95 \%$ confidence interval of the scores (binomial distribution). the PLMs. The experiments described below contribute to make such a hypothesis unlikely.

The length of the PLMs was examined in mutants with altered body length (Fig. $7 a$ ) as well as in $u n c-53$ and body length double mutants (Fig. $7 b$ ). For practical reasons, in particular their better observability, the analysis was confined to the PLMs.

At hatching, the length of the body of both $d p y-3(e 27)$ and lon-1(e185) mutants is very similar to wild-type length, but differences in postembryonic growth patterns result in large differences of length in adults (Fig. 7a). In both mutants the length of the PLMs is proportional to the length of the body and they stop at the same relative position in the animal, shortly anterior to the vulva, as they do in the wild type. This indicates that the length of the PLMs, at least during postembryonic development, is not fixed in a cell-intrinsic manner but varies with the length of the body.

Results described below suggest that the mechanism regulating the length of the PLMs in relation to the body during postembryonic development appears to be still operating in $u n c-53$ mutants. We determined the extension index in double mutants carrying unc-53(n166) as well as a mutation affecting body length. In both the shorter and the longer animals, the PLMs were observed to stop at the same relative position of the body as in animals carrying the $u n c-53$ mutation alone (Fig. $7 b$ ). In absolute length, therefore, the PLMs in the short double mutants are much shorter, and in the long double mutant much longer, than in animals carrying $u n c-53(166)$ alone. If the shortening of the PLMs in unc-53 animals was due to an intrinsic inability to synthesize structural components, one would expect the PLMs to grow always to the same length, irrespective of the length of the body. This length would be the maximum length they can achieve under the pathological conditions produced by the unc53 mutation. It is therefore surprising that they succeed in growing more in long double mutants, and that in dumpy double mutants they don't reach the length they would normally achieve in $u n c-53$ mutants of wild-type body shape. The lon and $d p y$ mutations affect the shape (long and thin for lon, and short and fat for $d p y$ ) rather than the size of the worm and it seems unlikely
Figure 7. $a$, PLM length, adult posterior body length (from the vulva to the start of the tail spike), and total body length at hatching, in wild type and in mutants with altered body length (see Materials and Methods for description of the measurements). For each genotype 30 animals were examined for PLM anatomy. In each case all PLMs were found to have wild-type anatomy, as defined by termination anterior to the vulva, in the region of the ALM cell body. $b$, PLM extension index, adult posterior body length, and total body length at hatching in strains carrying unc-53(n166) as well as mutations altering body length. The PLM extension indices were scored as described in Material and Methods, and in the Figure 6 caption. The measurements are given $\pm 1 \mathrm{SD} ; 95 \%$ confidence limits are derived from the binomial distribution.

\begin{tabular}{|c|c|c|c|c|}
\hline Genotype & Phenotype of Posterior Body & $\begin{array}{l}\begin{array}{l}\text { Posterior } \\
\text { body } \\
\text { length } \\
\text { (microns) } \\
(+/ \text { - s. d.) }\end{array} \\
\end{array}$ & $\begin{array}{l}\text { Total body } \\
\text { length } \\
\text { at hatching } \\
\text { (microns) } \\
\text { (+l- s.d.) }\end{array}$ & \begin{tabular}{|c|} 
PLM \\
extension \\
index (\%) \\
(95\% \% \\
coridence \\
limitis)
\end{tabular} \\
\hline a & & & & \\
\hline wild type & $\Longleftarrow$ & & & \\
\hline lon-I(e185) & $\Longleftrightarrow$ & $609 \pm 76$ & $206 \pm 12$ & - \\
\hline dpy-3(e27) & $\approx-10$ & $273 \pm 35$ & $193 \pm 14$ & - \\
\hline \multicolumn{5}{|l|}{ b } \\
\hline unc-53(n166) & $\Longleftrightarrow$ & $402 \pm 60$ & $193 \pm 15$ & $\begin{array}{c}44 \\
(35-54)\end{array}$ \\
\hline $\begin{array}{l}\text { unc-53(n166) } \\
\text { lon-1(e185) }\end{array}$ & $\Longleftarrow$ & $482 \pm 51$ & $202 \pm 15$ & $\begin{array}{c}42 \\
(30-55)\end{array}$ \\
\hline $\begin{array}{c}\text { unc-53(n166) } \\
d p y-3(e 27)\end{array}$ & $\Longleftarrow$ & $255 \pm 39$ & $192 \pm 17$ & $\begin{array}{c}53 \\
(43.64)\end{array}$ \\
\hline
\end{tabular}



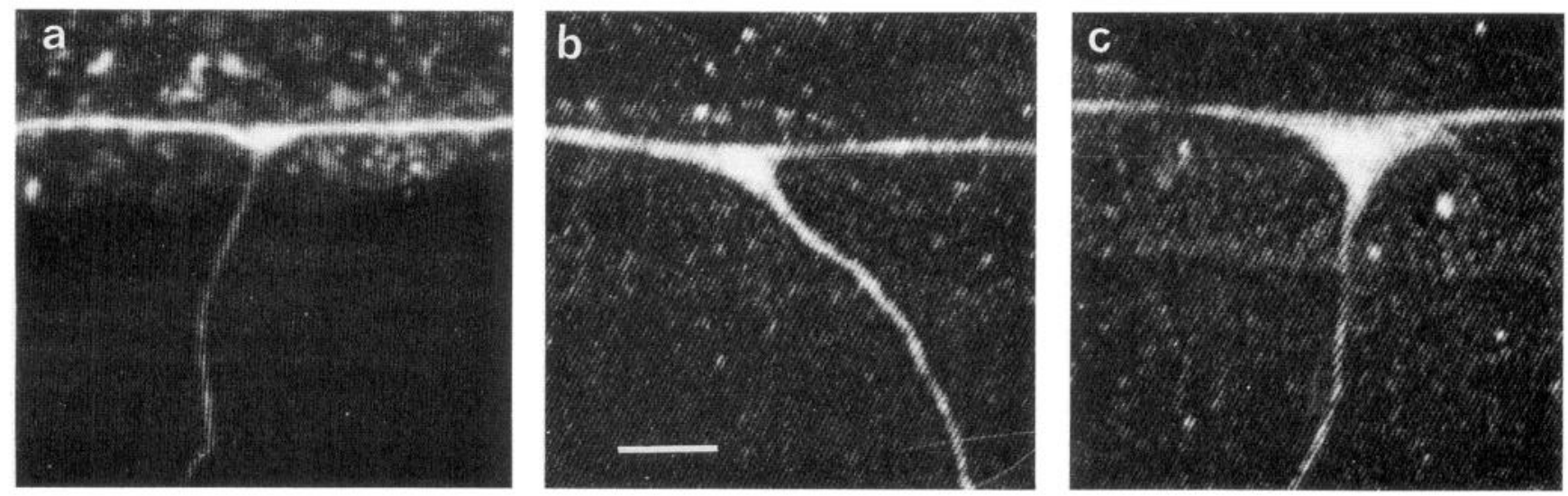

Figure 8. Branches of PLMs in unc-53 mutants. $a$, A branch made at the wild-type position in the region of the vulva and displaying a wild-type general morphology with a small diameter and a small branching point. $b$, A branch with an abnormal morphology of intermediate severity: the diameter of the branch is substantially enlarged but the branching point resembles the wild type. $c$, A branch with a severely abnormal branching point. Scale bar, $3 \mu \mathrm{m}$.

that these mutations would alter the efficiency of biosynthetic or metabolic processes precisely in such a way as to compensate for their influence on body shape.

The results described in this section reinforce the suggestion that $u n c-53$ is involved in spatially localized interactions, as mutations appear to prevent elongation into particular sections of the body rather than elongation per se.

\section{Placement and ultrastructure of MC branches}

PLM branches. In the wild type, the PLMs make a single branch directed into the ventral nerve cord, generally posterior to the vulva. In all unc-53 mutants examined, a significant fraction of the PLMs never reach the region of the vulva where the branch would be made. In spite of this, a branch was frequently found to be made at a more posterior position than in wild type and, in rare cases, more than one ectopic branch could be observed (Table 2).

In addition to being abnormally placed, mutant branches also have an abnormal morphology. The wild-type branches appear as very thin inconspicuous structures compared to the main process. In contrast, the majority of mutant branches had an aspect that was much more similar to that of the main process (Figs. 3, 8). In addition, the branching points were found to be enlarged (Fig. $8 b$ ), often severely $(8 c)$. The degree of abnormality of the branches was variable, and in some cases was extremely abnormal.

To study the distribution of the position and the morphology of the branches along the longitudinal axis, we examined the PLMs of unc-53(e2432) mutants. $e 2432$ is a weak allele in which the length of the PLMs is highly variable. The length of the PLMs and the position and morphology of the branches were scored (Fig. 9). Three characters where considered: the region of termination, the region in which the branch was positioned, and the morphology of the branch. Positions of end points and branches were scored by dividing the posterior body into three approximately equal regions. The most posterior region included the PLM cell bodies and the most anterior included the vulva (Fig. 9). Only those PLMs whose termination point and branching point could be visualized were scored. The morphology of the branches was scored subjectively as similar to the wild type,

Table 2. Frequency of branches in unc-53 mutants

\begin{tabular}{|c|c|c|c|c|c|c|}
\hline & \multirow{2}{*}{ Wild type } & \multicolumn{5}{|l|}{$u n c-53$} \\
\hline & & $e 2432$ & $n 569$ & $n 166$ & $n 152$ & $e 404$ \\
\hline $\begin{array}{l}\text { PLM with one } \\
\text { abnormal branch }\end{array}$ & $\begin{array}{l}0 / 170 \\
(0.0 \%)\end{array}$ & $\begin{array}{l}273 / 467 \\
(58.5 \%)\end{array}$ & $\begin{array}{l}105 / 185 \\
(56.8 \%)\end{array}$ & $\begin{array}{l}69 / 132 \\
(52.3 \%)\end{array}$ & $\begin{array}{l}69 / 136 \\
(50.7 \%)\end{array}$ & $\begin{array}{l}43 / 107 \\
(40.2 \%)\end{array}$ \\
\hline $\begin{array}{l}\text { PLM with multiple } \\
\text { abnormal branches }\end{array}$ & $\begin{array}{l}0 / 170 \\
(0.0 \%)\end{array}$ & $\begin{array}{l}10 / 467 \\
(3.9 \%)\end{array}$ & $\begin{array}{c}8 / 185 \\
(4.3 \%)\end{array}$ & $\begin{array}{l}2 / 132 \\
(1.5 \%)\end{array}$ & $\begin{array}{l}0 / 136 \\
(0.0 \%)\end{array}$ & $\begin{array}{l}1 / 107 \\
(0.9 \%)\end{array}$ \\
\hline $\begin{array}{l}\text { Abnormal ALM } \\
\text { branches vs total } \\
\text { number of observed } \\
\text { branches }\end{array}$ & $\begin{array}{l}0 / 120 \\
(0.0 \%)\end{array}$ & $\begin{array}{c}9 / 121 \\
(7.4 \%)\end{array}$ & $\begin{array}{c}2 / 54 \\
(3.7 \%)\end{array}$ & $\begin{array}{c}2 / 11 \\
(18.2 \%)\end{array}$ & $\begin{array}{c}5 / 27 \\
(18.5 \%)\end{array}$ & $\begin{array}{l}13 / 18 \\
(72.2 \%)\end{array}$ \\
\hline
\end{tabular}

The PLM data represent the frequency of PLMs possessing one or multiple abnormally enlarged branches, in five unc53 alleles and in the wild type. The alleles are tabulated from left to right in the order of increasing severity in regard to PLM length (see Fig. 6). A decrease in the incidence of branches in alleles of increasing severity can be observed and might be attributed to a decrease in the length of the main process and the resulting decrease in the opportunity to produce a branch. The ALM data represent frequency of abnormal versus normal ALM branches. In the weaker alleles in which the majority of the ALMs extend to or beyond the normal position of the branch, the majority of the branches are normal in position and morphology. In the stronger alleles in which a higher proportion of the ALMs stop before the nerve ring, branches were only rarely observed but were frequently abnormal. 


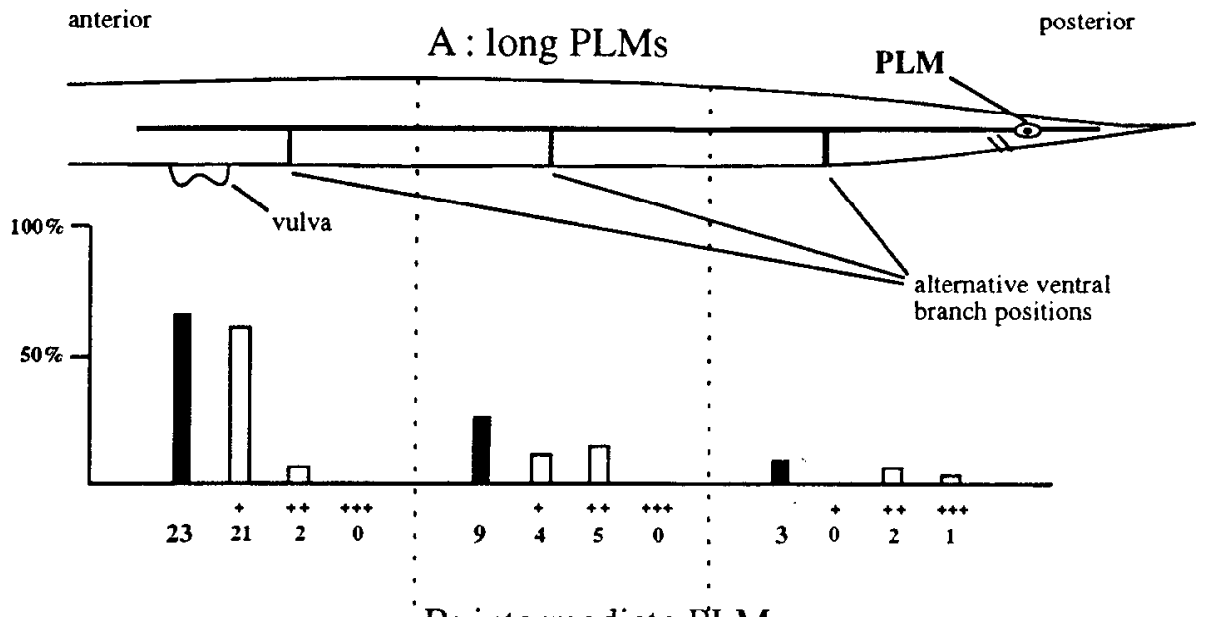

Figure 9. Placement and morphology of the ventral branch of PLMs in unc53(e2432) mutants. e2432 is a weak allele in which PLMs of almost wild-type length as well as short PLMs can be observed. The posterior body was conceptually divided into three approximately equal regions, the most posterior starting at the PLM cell bodies and the most anterior including the vulva. The PLMs were classified according to the region in which they stopped $(A$, long PLMs; $B$, intermediate PLMs; $C$, short PLMs). For each of the three classes of PLM, the position and morphology of observed branches were scored separately. The position was scored according to the same three regions as PLM termination and the morphology of the branches was scored subjectively as wild type $(+)$, intermediate $(++)$, and very abnormal $(+++)$. The criteria for abnormality included the branch diameter and the degree of enlargement of the branching point (see Fig. 8). The solid bars give, for each category of PLMs and for each region, the percentage of branches being made in that region. The open bars correspond to a breakdown of the adjacent solid bar according to the three morphological categories of branches. The numbers under each bar correspond to the actual number of branches observed in each case.

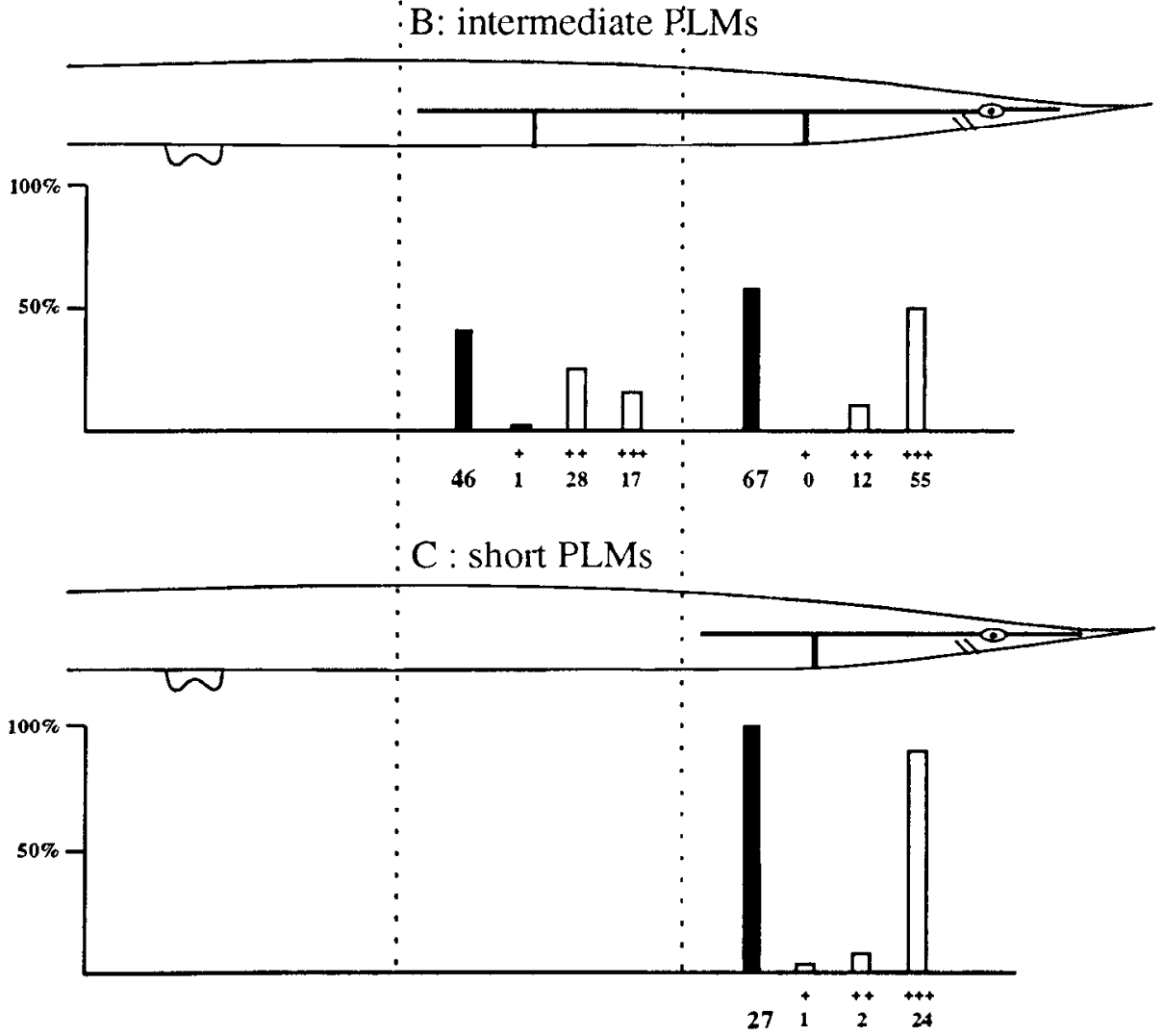

intermediate, and very abnormal. The criteria for abnormality included the branch diameter and the degree of enlargement of the branching point (Fig. 8).

In the case of those PLMs that reach the most distal region, which includes the vulva, the majority of branches were also made in this region (Fig. 9 , top). In contrast, in the case of those PLMs that stop in the middle region, the distribution of branches appeared much more even throughout the two regions over which these PLMs extended. The occurrence of slightly more branches in the most posterior region is due to the coarseness of the scoring. A PLM that extends for only a very short distance into the middle region will be scored as being of intermediate length but will have no real opportunity to make a branch in the middle region. Table 2 shows that though a high proportion of mutant PLMs possess a branch, there is a decrease in the incidence of branches in alleles of increasing severity in terms of the length of the PLMs (see Fig. 5). Together, the abovc observations might suggest that in the region of the vulva a localized signal induces the PLMs to branch. In the absence of this inducing signal, branches are made at random, with the probability of making a branch depending on the length of the main process.

The morphology of the branch was scored as a function of the position of the branch (Fig. 9, open bars). This revealed a correlation between position and morphology, with the morphologies becoming more abnormal with more posterior positions. There was no close correlation, however, between branch morphology and the length of the PLMs: even in long- or intermediate-length PLMs the branch could be very abnormal when it occurred in the most posterior region.

Branches of other MCs. In all unc- 53 alleles studied, the ALMs extend at least to the pharyngeal-intestinal valve, a point very 


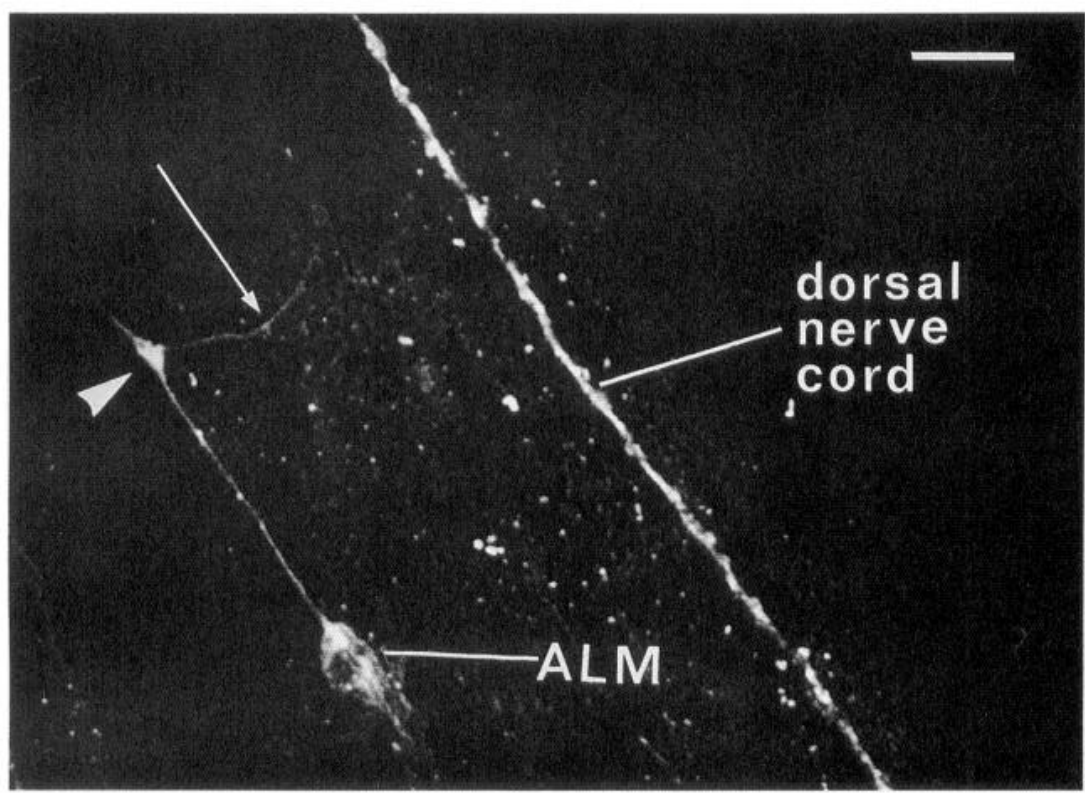

Figure 10. An ALM in an unc53(e404) mutant with an ectopic dorsal branch (arrow) close to the cell body. The " $A L M$ " label points to the ALM cell body. An arrowhead points to the abnormally large varicosity at the branching point. Scale bar, $10 \mu \mathrm{m}$.

close to where a branch directed into the nerve ring occurs in the wild type (see Figs. 3-5). Indeed, in weaker unc-53 alleles a large majority of observed branches were made at this location (Table 2). Branches at the wild-type position were frequently difficult to observe in wild-type as well as in mutant animals, because of the intense staining of the nerve ring. However, when mutant branches were localized at the wild-type position and could be properly visualized, they appeared invariably to have a wild-type morphology. In rare cases (Table 2), branches were observed to be made dorsally instead of into the nerve ring and at much more posterior positions, close to the ALM cell body (Fig. 10). Such dorsally directed branches frequently resembled the most abnormal PLM branches. In the more severe alleles, producing the most extreme ALM shortening, there is a high proportion of abnormal branches. Such branches form the majority in the most severe allele, $e 404$ (Table 2).

The situation with the ALMs therefore resembles that observed with the PLMs: when an ALM is long enough to reach the level at which branches normally occur, then a branch is made at that location (the nerve ring) and it is of normal morphology. Normal branches are, however, observed much more frequently than in the case of the PLMs, because in the weaker alleles the majority of ALMs are long enough to have the opportunity to branch at the wild-type location and do not produce ectopic branches.

As with the ALMs, AVM generally reached at least the level (nerve ring) at which the branch is normally made. However, the staining of the numerous nervous elements in this region generally prevented observation of the branch. The processes of the MC are stained more intensely than those of other neurons. For this reason, observation of AVM branches was sometimes possible in weakly stained preparations. In these cases the AVM branch mostly appeared to be made correctly into the nerve ring. Interestingly, in a total of eight cases observed in various alleles, the branch was found to be made substantially earlier, in the RVG or slightly posterior. These ectopic branches then ran in the ventral cord, parallel to the main process.

No branches of PVMs, which do not normally branch in the wild type, were observed. However, PVMs were always difficult to observe and frequently very short. A conclusion about the branching of this neuron in unc-53 mutants could therefore not be reached in this study.

Together, the observations of all the MCs support the hypothesis that the branches of the MCs are induced at specific locations by an external signal. When the signal fails because the cell's process does not reach the position at which the signal is provided, then the branches are made at random ectopic locations. In addition, the overall morphology of the ectopic branches appears to be altered in a graded manner as a function of position along the longitudinal axis, with the most posterior branches being the most abnormal.

Branch ultrastructure. The ultrastructure of the MCs has been investigated in detail in the past by others using EM (Chalfie and Thomson, 1979, 1982; Chalfie and Sulston, 1981; Chalfie, 1984; White et al., 1986). They display several specialized ultrastructural features that are required for sensory transduction (Fig. 11). In the wild type the main processes of the MCs contain a bundle of conspicuous 15-protofilament microtubules. The MCs are closely apposed to a darkly staining region of the cuticle. Since the LMs are located at levels of the body wall at which the hypodermis is thick, their apposition to the cuticle is made possible by the fact that they are embedded in a pocket within the hypodermis. Finally, the main process displays a specialized and polarized extracellular matrix, called the mantle, which fills the space between the axon and the cuticle. None of these ultrastructural specializations are shared by the branches (Fig. 11).

The ectopic branches of the PLMs in 22432 (two branches) and in $e 404$ (two branches) mutants were examined by EM. They all displayed the ultrastructural features of the main process, including large diameter and straight course, embedding in the hypodermis, large darkly stained microtubules, and an asymmetric mantle correctly positioned on the side of the cuticle (Fig. 12). The main difference from the main process was that they were not very closely apposed to the cuticle, nor was their presence associated with increased local staining of the cuticle.

The number of animals that can be examined by EM is limited, and no normally placed branches, which are rare in the mutants, could be found and examined. For the same reason, 
Figure 11. Electron micrograph showing a transverse section of a PLM at the level of the ventral branch in a wild-type animal. The various specializations of the MCs can be seen. They include close apposition to the cuticle and an associated dark region, location in a pocket in the hypodermis, the presence of an abundant extracellular matrix (the mantle) in the pocket on the side of the cuticle, and large darkly staining microtubules (arrowheads). The branch entirely lacks these specializations. It leaves the pocket dorsally and travels on the surface of the hypodermis to join the ventral cord. Magnification, $150,000 \times$.

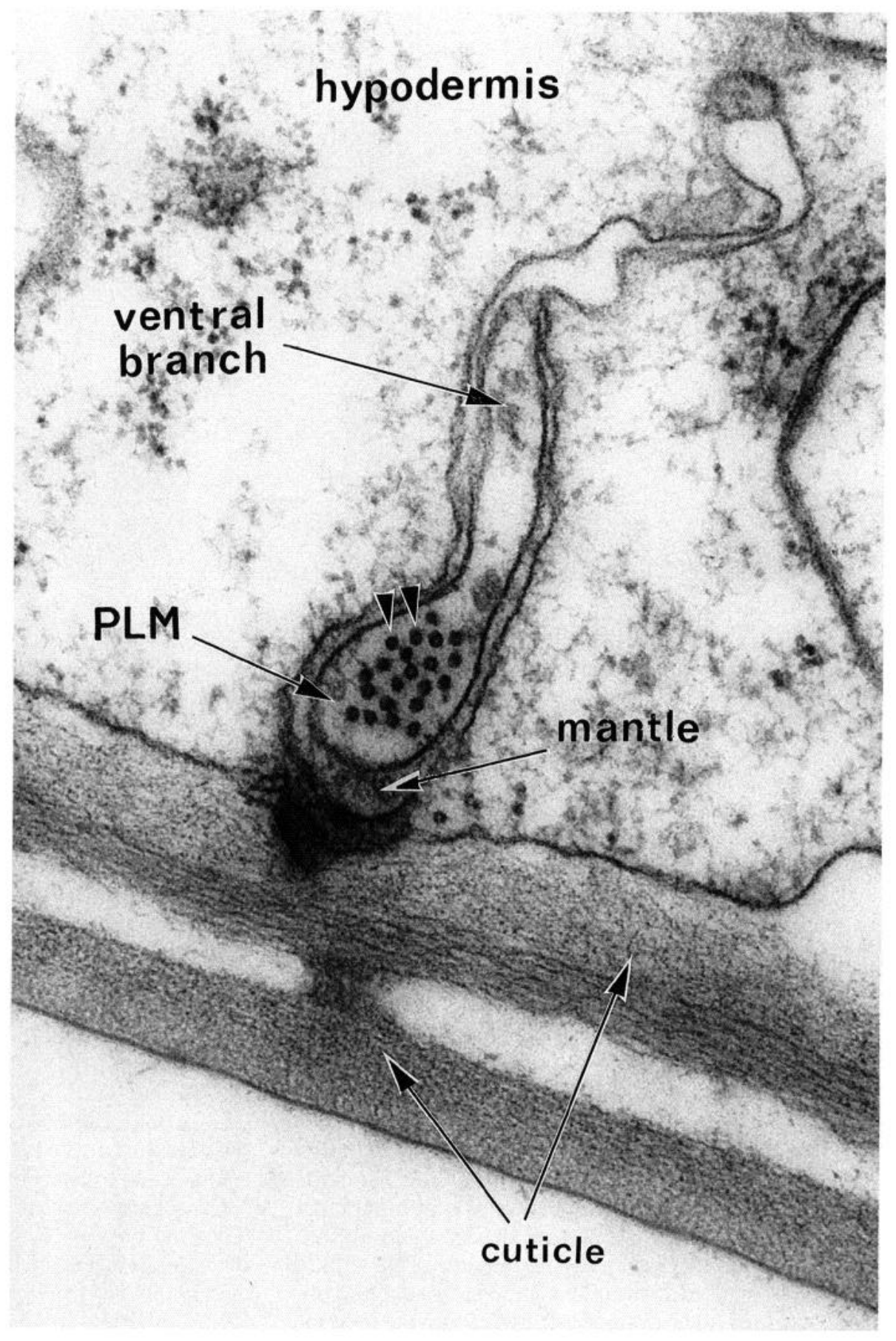

we also did not observe any ectopic ALM branches. The vast majority of ALM branches in the mutants appear normally placed and of normal diameter, and this was also the case in eight ALM branches examined by EM in e2432 mutants (data not shown).

Surprisingly, in some cases the bundle of microtubules in the branch appeared not to derive from the main bundle by splitting, but appeared perfectly perpendicular to the bundle in the main process. The two bundles apparently interpenetrate each other at right angles (Fig. 12).

In summary, the relative enlargement of the ectopic branches observed by immunocytochemistry is accompanied by a series of qualitative changes that make the branch similar to the main process. The observations suggest that the wild-type branch with its absence of specializations is indeed a particular domain of the neuron, which is produced or maintained only when the branch is induced at its normal location.

\section{Interactions during extracellular matrix synthesis}

The ectopic branches, as well as some of the misguided main processes of the PLMs that reach the ventral cord, continue within it for variable distances. Both ectopic branches and misplaced main processes display the same set of features during their trajectory in the ventral cord. Instead of remaining in a particular location relative to other processes as is the rule for processes in the ventral cord (White et al., 1986), they constantly 


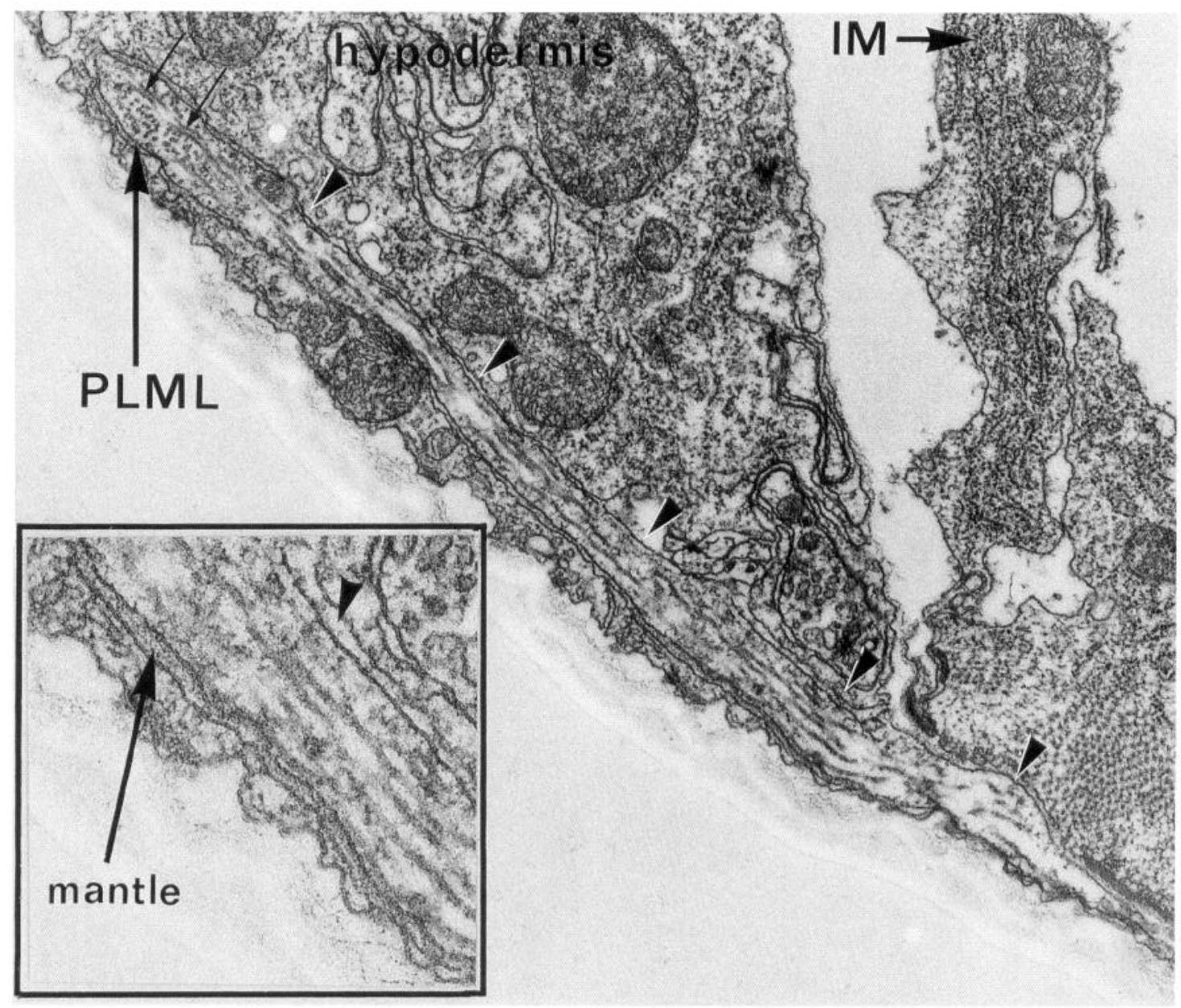

Figure 12. Electron micrograph of a transverse section of a left PLM at the level of a ventral branch in an unc-53(e404) mutant. The posterior location of the branching can be inferred from the presence of the intestinal muscle $(I M)$, which is located at the posterior end of the gut. The "PLML" label points to the main process that runs perpendicular to the plane of the figure. The branch issuing from it is indicated by a series of arrowheads in the main picture. In contrast to branches in the wild type (see Fig. 11), the mutant branch closely resembles the main process. The mutant branch has a diameter comparable to that of the main process, it is embedded in the hypodermis, it contains large darkly stained microtubules (the small arrows point to microtubules that start in the main process), and it is associated with a mantle restricted to the side of the cuticle (see inset). The arrowhead in the inset points to the extracellular space on the internal side that is devoid of mantle material. The main difference from the main process is that the mutant branch is not as closely apposed to the cuticle, which correspondingly fails to show a specific darkening. The microtubule bundle in the branch appears to be perpendicular (small arrows) to the microtubules of the bundle in the main process, which appear as dark dots. PLML, posterior lateral microtubule cell left. Magnification, 50,000×.

change position relative to the other neurons and the hypodermis. They are sometimes associated with the hypodermis on the side of the cuticle, sometimes with the ventral hypodermal ridge, and sometimes entirely surrounded by neurons. Whenever they are in contact with the hypodermis they display a mantle along the surface of contact (Fig. 13c,d), but are completely devoid of a mantle when no contact is made with the hypodermis (Fig. 13a,b). This strongly suggests that mantle synthesis requires an interaction between the PLM axon and the hypodermis.

In contrast to the ectopic PLMs, PVM and AVM processes remain correctly positioned at the bottom of the ventral cord in close apposition to the cuticle and they display an uninterrupted mantle (not shown). This suggests that the mobility of the ectopic PLMs in the ventral cord might be due to a lack of guidance cues, as the PLMs' normal trajectory does not include the ventral cord. It also suggests that the mantle by itself is not sufficient to trap MCs at the bottom of the ventral cord by attaching them to the hypodermis, but that more specific guidance cues are involved.

\section{Discussion}

The role of unc-53 during development

Three sets of observations suggest that the gene unc-53 plays a role in the mechanisms that regulate the length and the placement of axons. First, whole process bundles are misguided in the mutant, which suggests that unc-53 mutations affect pioneering neurons. Second, there is a spatially restricted requirement for unc-53 activity. Third, the mechanism by which mutations in unc-53(n166) prevent the PLMs from extending processes is not by impairment of their biosynthetic abilities.

unc-53 mutations affect pioneering neurons. Axonal tracts are believed to be established by pioneering neurons able to follow diffusible or substrate-bound guidance cues (reviewed by Hortsch and Goodman, 1991; Reichardt and Tomaselli, 1991; TessierLavigne and Placzek, 1991). Axons that grow out subsequently 

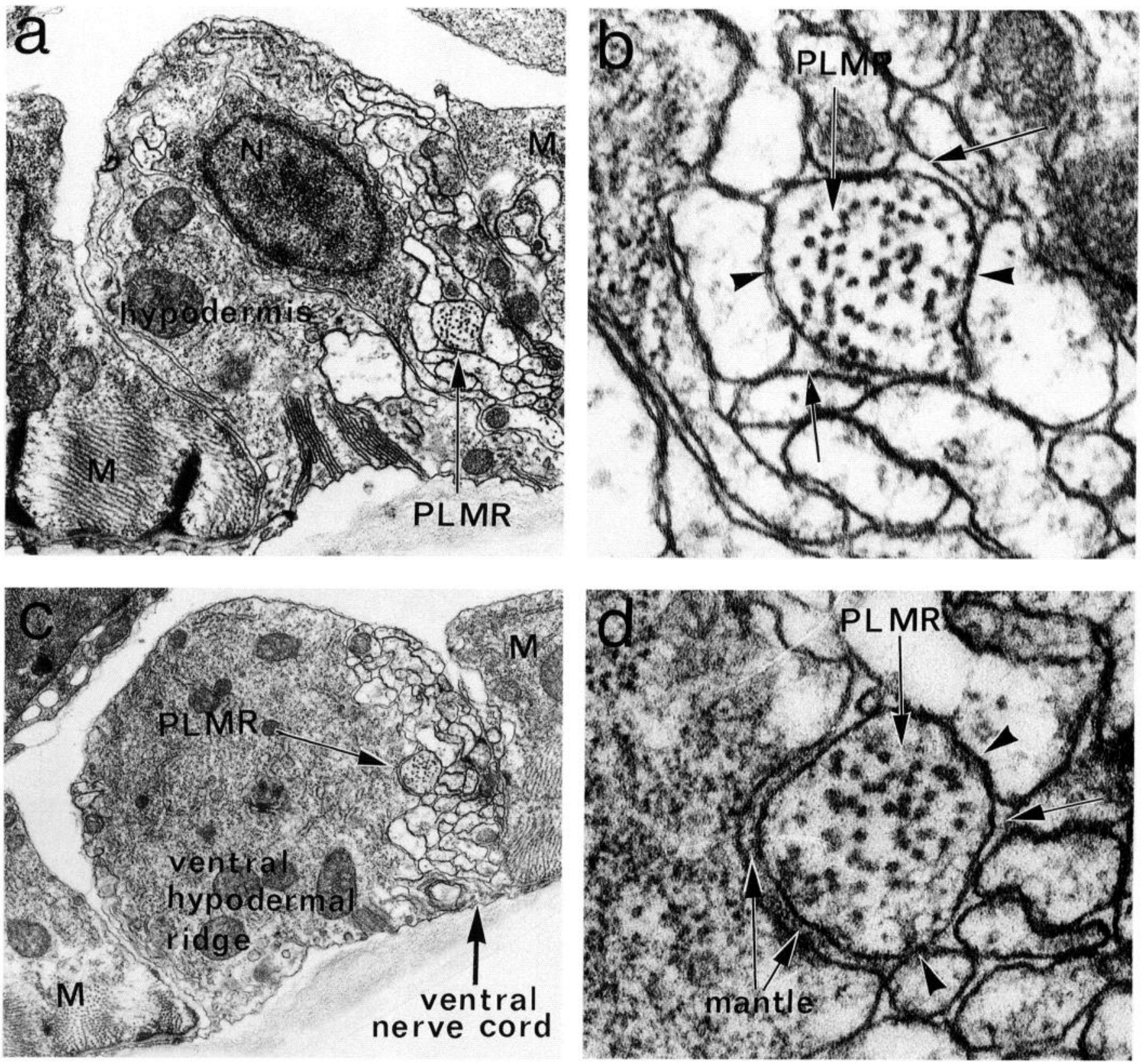

Figure 13. Electron micrographs of a transverse section of the ventral cord of an unc-53(e2432) mutant. When morphologically abnormal branches reach the ventral cord, they run in it, sometimes for considerable distances, but they do not remain confined to a given neighborhood. Instead, they change their position constantly, and apparently randomly, relative to other neurons and the hypodermis. $a$, An abnormal branch of a PLM in the ventral cord entirely surrounded by neurons. $b$, A further magnification of the same section as in $a$ shows the total absence of a mantle. Arrowheads point to the close apposition of the PLM's plasma membrane to that of other neurons, and the arrows show that the extracellular space between neurons and the PLM does not contain mantle material. $c$, The same neuron as in $a$ and $b$, approximately $10 \mu \mathrm{m}$ farther anteriorly in the ventral cord, is now in contact with the ventral hypodermal ridge. $d$, Further magnification of the same section as in $c$ shows the presence of a mantle on the side of the hypodermis. Arrowheads point to regions at which the PLM is in close contact with other neurons and where no mantle is found. The arrow points to a region of clear extracellular space devoid of mantle on the side of the other neurons. $M$, muscle; $N$, neuronal nucleus; $P L M R$, posterior lateral microtubule cell right. Magnification: $a$ and $c, 15,000 \times ; b$ and $d, 85,000 \times$.

form large process tracts by fasciculating with the pioneering neurons (e.g., Kuwada, 1986; Klose and Bentley, 1989). There are indications that such a succession of events might also be the rule in C. elegans (Durbin, 1987; Walthall and Chalfie, 1988; Hedgecock et al., 1990). In unc-53 mutants, entire process bundles can be observed to be misguided. For example, the sublateral nerve cords and the left ventral cords become misplaced without losing their coherence as a bundle (Figs. 1, 2), which suggests that unc-53 affects the guidance of the neurons that pioneer these process tracts, but not the ability of neurons extending later to fasciculate with the misguided pioneers.

Moreover, the LMs are strongly affected by unc -53 mutations and various considerations suggest that they are pioneering neurons. In the mature nervous system each LM is associated with only one other process: each ALM with an LM anterior lateral neuron (ALN), and each PLM, with a posterior lateral neuron (PLN). These neurons could conceivably have pioneered the paths of the MC. The embryonic birth of the ALNs occurs, 
however, substantially later than that of the ALMs (Sulston et al., 1983). In addition, their cell bodies are situated in the lumbar ganglia behind the anus, at a considerable distance from the ALMs. It is unlikely, therefore, that their processes could have reached the ALMs at the time of their outgrowth. Furthermore, in EM reconstructions of embryos during the time of axonal outgrowth (Durbin, 1987), no processes were seen to be associated with the ALMs. The situation is even clearer for the PLN neurons, which are born during postembryonic development (Sulston and Horvitz, 1977) and therefore cannot contribute to the development of the embryonic PLMs.

Spatially restricted requirements for unc-53. The PLMs require wild-type $u n c-53$ activity for their entire outgrowth. In the strongest allele (e404), there can be an almost complete absence of anterior outgrowth of thcsc cclls. In addition, in the weakest alleles the outgrowth is substantial but nonetheless invariably stunted, which indicates a dependance on $u n c-53$ for all parts of the outgrowth. Due to the difficulties in observing the ventral microtubule neurons with our methods, the pattern of penetrance and expressivity of PVM could not be determined in the same detail as that of the PLMs. However, it is clear that PVM's requirement for unc-53 starts immediately after outgrowth, as it can be almost abolished in the stronger alleles. In weaker alleles some outgrowth of PVM is observed, but outgrowth beyond the vulva was almost never observed. In stark contrast to the situation of the posterior MCs, the outgrowth and guidance of the ALMs and AVM appear to require unc-53 activity only in the head, the most distal part of their normal extension. Even in the strongest alleles, the level of the pharyngeal intestinal valve is always reached.

In addition to defects in the nervous system that presumably interact with the basal face of the hypodermis, unc-53 mutants display defects in the external cuticle (S. Hekimi and D. Kershaw, unpublished observations), which is synthesized by the hypodermis on its apical face. It is likely, therefore, that the hypodermis expresses $u n c-53$, and it is tempting to speculate that the spatially restricted requirement for the activity corresponds to a physical localization of the gene product on the body wall, in the posterior body, and in the head. Alternatively, the $u n c-53$ gene product might be more uniformly distributed but is required only in certain regions through combinatorial effects involving other gene products.

Specificity of unc-53 action. We have observed that the absolute length of the PLMs depends on the length of the body (Fig. 7). They are shorter than wild type in short worms ( $d p y$ 3 mutants) and longer in long worms (lon- 1 mutants). It indicates that the PLMs in worms that arc not mutant for $u n c-53$ have the capacity for increased growth in comparison to the wild type. In unc-53(n166) mutants that also carry mutations modifying body shape, this translates into PLMs that are either much shorter or much longer than the PLMs of animals carrying $n 166$ alone. These observations strongly oppose a model where a nonspecific pathological condition in unc-53 mutants stunts the growth of the PLMs through an impairment of their capacity to synthesize their axonal process. With such a model one would expect the PLMs to reach always at least the size they reach in animals carrying $u n c-53$ alone. Although it appears unlikely that such a pathological condition could be exacerbated or cured by the $d p y$ and lon mutations precisely in a way compensatory for their particular influence on body shape, the possibility of cellintrinsic interactions between $u n c-53$ and the test mutations is not excluded by our experiments.
It remains possible that $u n c-53$ mutations induce pathology in PLMs only during embryogenesis. Our experiments would then not be conclusive, as the mutants we have examined have a normal body shape at the end of embryonic development. Mutants with an altered pattern of embryonic elongation have been described (Brenner, 1974; Priess and Hirsh, 1986) and could be examined in an analogous fashion. However, they would require reconstructions based on electron micrographs of serial sections, as the resolution of our methods is not sufficient for unambiguous examination of early larvae.

unc-53 affects longitudinal tracts. The unc-53 mutations we have examined affect most or all longitudinal process tracts, including the ventral, dorsal, and sublateral nerve cords and the four LMs. The status of two longitudinal tracts, the process of ALN while alone on the posterior lateral hypodermis, and the bundle of processes associated with the canals of the excretory cell could not be determined because these axons are usually only faintly stained and ectopic processes frequently run in these locations. In addition to our observations, Hedgecock et al. (1987) reported that the long posterior canals of the excretory cell were shortened in unc-53(e404). The pattern of defects observed suggest, therefore, that $u n c-53$ specifically affects axonal guidance in the longitudinal axis. However, we have not rigorously established that it has no effect on circumferential guidance. Though we have not identified any defects in the dorsal commissures, these were not examined as individually identified processes. In general, in any given animal, only a few commissures could be unambiguously observed throughout their length. Missing commissures or commissures subtly misplaced in relation to the others would not have been identified. Our observations are confirmed in part by a recent study (McIntire et al., 1992) of mutations affecting neuroanatomy. These authors failed to identify $u n c-53$ in a survey of uncoordinated mutants for which they used immunocytochemical detection of the neurotransmitter GABA to visualize a subset of neurons including neurons with circumferential trajectories. It suggests that circumferential pathfinding is at least not globally affected and underscores the fact the unc-53 mutations appear to affect a discrete set of neurons and mechanisms.

Further elucidation of the functions and the mechanisms of action of $u n c-53$ will depend on its molecular characterization, and on the investigation of the interactions of $u n c-53$ with the other genes that have been shown to be involved in shaping the neuroanatomy of the worm (Hedgecock et al., 1990; McIntire et al., 1992).

\section{Cell-extrinsic mechanisms in $M C$ differentiation}

Our observations on the MCs in unc-53 mutants suggest the involvement of a number of cell-extrinsic cues in the differentiation of a variety of features of these neurons. These features include the length and the placement of processes, the position of branches, the subcellular morphology of branches, and the production of a specialized extracellular matrix.

Length of processes. In an organism with such a highly stereotyped development as $C$. elegans, participation of cell-autonomous mechanisms in the determination of axonal length cannot be excluded. However, our observations on the MCs in $d p y$ and lon mutants and the general observation that mutants with altered body shape frequently display normal behavior suggest that the dimensions of neurons are actively adjusted to the dimensions of the body they innervate.

Various mechanisms have been proposed to account for the 
regulation of axonal length. For example, the targets of axonal projections could participate in the regulation of their length in at least two ways, either by being the source of an attractant involved in guiding the axon toward it, or by being a stop signal for axons guided by other means. Mclntire et al. (1992) observed that the processes of hermaphrodite-specific neurons (HSNs), in egl-43 mutants in which the cell bodies of the HSNs fail to migrate anteriorly, extend for a longer distance to reach their target in the nerve ring. This appears to be a clear case of target control and given the large distances involved is more likely to represent a case of the target providing a stop rather than an attractive signal to the HSN.

The involvement of $u n c-53$ in the development of the MCs is apparently of a different kind. The fact that an allelic series of expressivity can be constructed using an index of extension suggests that the amount of unc- 53 activity is positively correlated with the length to which the axons will grow (Fig. 6). This is not consistent with $u n c-53$ being involved in an attractive signal emanating from a localized source, unless it were involved in the localization of the signal. A decrease in the intensity of an attractive signal would produce a threshold effect resulting in either a complete absence of guided outgrowth or normal outgrowth as long as the threshold is reached. For $u n c-53$ to be involved in a diffusible message, one would have to postulate that it is involved in a graded repulsive signal whose intensity is highest in the region where the outgrowth starts and drives the growth cone forward until its intensity decreases sufficiently. To be sufficiently precise such a mechanism might have to be linked to a stop signal from the target. Such a model could account for the observed link in the allelic series between the length of axons and the degree to which their placement is perturbed, which suggests a common underlying developmental mechanism. If the inhibitory activity is highest around the cell body and decreases with the distance away from it, for example, as it would in a tubular worm if the signal were a diffusible substance, the guidance of the axon would result from the fact that the inhibition decreases most rapidly along the longitudinal axis.

Another possible model that would account for our observations is based on in vitro studies in other systems that have demonstrated that tension applied to axons may act as a stimulant of axonal growth (Bray, 1984; Dennerll et al., 1988; Heidemann and Buxbaum, 1990). It has also been shown that growth cones pull on the substratum (Lamoureux et al., 1989) and generate tension in the axon (Bray, 1979). In C. elegans, many neuronal processes grow out during the period of embryonic elongation (Durbin, 1987), which is a major morphogenetic event involving the circumferential compression of the ovoid embryo by specialized structures in its own epidermis and resulting in its elongation into the shape of a worm (Priess and Hirsh, 1986). During this period, a large amount of tension will be generated along the longitudinal axis of the embryo. Pioneering axons may therefore grow preferentially along the lines of maximum tension, with their final extension being determined by the duration of the perceived tension and the strength of the axons' adhesion to the substratum. In such a model the product of $u n c-53$ could simply be involved in the adhesion of the MCs to the hypodermis. Mutations in $u n c-53$ would result in less adhesion and therefore in less tension being transferred to the axon, resulting in a decrease of its growth rate.

Our results imply that the product of $u n c-53$ is involved in the mechanisms that allow body shape to impinge on the shape of the MCs. However, they don't imply that unc-53 is not expressed in the MCs themselves. For example, the adhesion model outlined above could involve homophilic adhesion between unc-53 molecules in the hypodermis and in the MCs.

Placement of processes. As described in Results, in the absence of normal unc-53 activity the PLMs lose their ability to run in a straight line and tend to turn ventrally but never dorsally. Again, it seems likely that this preference might be the result of some cell-extrinsic guidance cues normally masked by the action of $u n c-53$, rather than the result of some intrinsic and cellspecific handedness in the path-finding properties of the PLMs. It is generally believed (Hedgecock et al., 1990; McIntire et al., 1992) that the guidance of all ventral trajectories in the worm requires the products of the genes $u n c-6$ and $u n c-40$. However, we have made preliminary observations in double mutants carrying $u n c-53$ as well as $u n c-6$ or $u n c-40$ indicating that the ventral turn of the PLMs and the ventral placement of their branches do not depend on unc- 6 and $u n c-40$.

Synthesis of the mantle. The mantle is a specialized extracellular matrix between the MCs and the hypodermis, normally on the side of the cuticle (Fig. 11). It is not known if the mantle is synthesized by the hypodermis or by the MCs. Our observation of the ventral cord of unc-53 animals (Fig. 12) indicates that the synthesis of the mantle requires a close contact between the hypodermis and the neurons, presumably because of contact-mediated inductive interactions.

Placement of branches. Chalfie et al. (1983) have shown that the branch of the AVM cell is induced by a localized signal. They found that no branch was formed when AVM was prevented from receiving the signal. We reached a similar conclusion for the formation of the branches of PLMs at their wildtype position. However, we find that even when the inductive signal could not be perceived by the PLMs because they are too short to reach it, a number of them will nonetheless branch at random locations (Table 2). The difference between these observations could be due to a number of factors, such as an intrinsic difference between AVM and the PLMs, or a sampling effect due to the small number of mispositioned AVMs examined by Chalfie et al. (1983). Finally, we cannot exclude an indirect effect of the $u n c-53$ mutation. For example, the $u n c-53$ background could reveal hidden cues (such as a branching signal), as hypothesized above to account for the ventral turns of the main process.

Localized inductive signals for the positioning of branches might be a common mechanism in C. elegans. At least one other neuronal cell type, the $\mathrm{VC}$ motor neurons that innervate the vulval musculature, and possibly the HSNs as well, has been shown by $\mathrm{Li}$ and Chalfie (1990) to receive inductive cues from the vulval cells for branch position.

Morphology of branches. The overall morphology of the PLM branches changes in a graded manner along the anteroposterior axis, with the wild-type morphology representing the extreme at the anterior end of this gradient (see Figs. 8, 9). The severity of these morphological features is therefore correlated with proximity to the cell body. It could reflect the ease with which material synthesized in the cell body can be transported to distant sites. However, the fact that the morphology of the main process itself does not show any sign of changing over its course does not support this hypothesis. The severity of the phenotype of the branch also does not correlate with the total amount of process synthesized by the PLMs, as very early branches of long PLMs are as abnormal as those from very short PLMs (Fig. 9). 
These observations suggest, therefore, that graded interactions with the cellular environment are responsible for the gradual change in morphology.

EM examination of the ectopic branches showed that they displayed all the ultrastructural features of the main process (Fig. 12). This suggests that this set of features might always occur together in an all or none fashion. The presence of one of these features could be the trigger for the differentiation of the others. However, distinct mutations have been described in which either the mantle or the specialized microtubules are missing but all the other features are present (Chalfie, 1984). Neither of these two features appears, therefore, to act as trigger.

\section{Conclusion}

The analysis of unc-53 mutants has given insights into the way unc-53 influences normal nervous development, as well as into the mechanisms by which the differentiated features of a particular class of neurons arise. We showed that $u n c-53$ mutations affect the guidance of pioneering neurons and that longitudinal guidance of the processes of at least certain neurons is controlled in different ways in different regions of the worm. We present evidence for the involvement of cell-extrinsic mechanisms in the control of the length and placement of the main process of neurons. We also show that cell-extrinsic factors determine a number of other differentiated features, including the position of branches along the main axon, the presence of specialized microtubules in these branches, and the nature of the extracellular matrix surrounding them.

\section{References}

Bray D (1979) Mechanical tension produced by nerve cells in tissue culture. J Cell Sci 37:391-410.

Bray D (1984) Axonal growth in response to experimentally applied tension. Dev Biol 102:379-389.

Brenner S (1974) The genetics of Caenorhabditis elegans. Genetics 77: 71-94.

Chalfie M (1984) Genetic analysis of nematode nerve-cell differentiation. Bioscience 34(5):295-299.

Chalfie M, Au M (1989) Genetic control of differentiation of the Caenorhabditis elegans touch receptor neurons. Science 243:1027-1033.

Chalfie M, Sulston J (1981) Developmental genetics of mechanosensory neurons of Caenorhabditis elegans. Dev Biol 82:358-370.

Chalfie M, Thomson JN (1979) Organization of neuronal microtubules in the nematode Caenorhabditis elegans. J Cell Biol 82:278-289.

Chalfie M, Thomson JN (1982) Structural and functional diversity in the neuronal microtubules of Caenorhabditis elegans. J Cell Biol 93: 15-23.

Chalfie M, Thomson JN, Sulston JE (1983) Induction of neuronal branching in Caenorhabditis elegans. Science 221:61-63.

Dennerll TJ, Joshi HC, Steel VL, Buxbaum RE, Heidemann SR (1988) Tension and compression in the cytoskeleton II: quantitative measurements. J Cell Biol 107:665-664.

Driscoll M, Chalfie M (1991) The mec-4 gene is a member of a family of Caenorhabditis elegans genes that can mutate to induce neuronal degeneration. Nature 349:588-593.

Durbin RM (1987) Studies on the development and organization of the nervous system of Caenorhabditis elegans. PhD thesis, University of Cambridge, Cambridge, England.

Finney M, Ruvkun G (1990) The unc-86 gene product couples cell lineage and cell identity in C. elegans. Cell 63:895-905.

Hall DH, Hedgecock EM (1991) Kinesin-related gene unc-104 is required for axonal transport of synaptic vesicles in C. elegans. Cell 65: $837-847$.
Hamclin M, Scott IM, Way JC, Culotti JG (1992) The mec-7 betatubulin gene of Caenorhabditis elegans is expressed primarily in the touch receptor neurons. EMBO J 11:2885-2893.

Hedgecock EM, Culotti JG, Thomson JN, Perkins LA (1985) Axonal guidance mutants of Caenorhabditis elegans identified by filling sensory neurons with fluorescein dyes. Dev Biol 111:158-170.

Hedgecock EM, Culotti JG, Hall DH, Stern BD (1987) Genetics of cell and axon migrations in Caenorhabditis elegans. Development 100:365-382.

Hedgecock EM, Culotti JG, Hall DH (1990) The unc-5, unc-6, and unc- $\mathbf{4 0}$ genes guide circumferential migrations of pioneer axons and mesodermal cells on the epidermis in C. elegans. Neuron 2:61-85.

Heidemann SR, Buxbaum RE (1990) Tension as a regulator and integrator of axonal growth. Cell Motil Cytoskel 7:6-10.

Hckimi S (1990) A neuron-specific antigen in C. elegans allows visualization of the entire neryous system. Neuron 4:855-865.

Hodgkin J (1983) Male phenotypes and mating efficiency in Caenorhabditis elegans. Genetics 103:43-64.

Hortsch M, Goodman CS (1991) Cell and substrate adhesion in Drosophila. Annu Rev Cell Biol 7:123-145.

Klose M, Bentley D (1989) Transient pioneer neurons are essential for formation of an embryonic peripheral nerve. Science 245:982984.

Kuwada J (1986) Cell recognition by neuronal growth cones in a simple vertebrate embryo. Science 233:740-404.

Lamoureux P, Buxbaum RE, Heidemann SR (1989) Direct evidence that growth cones pull. Nature 340:159-162.

Li C, Chalfie M (1990) Organogenesis in C. elegans: positioning of neurons and muscles in the egg-laying system. Neuron 4:681-695.

McIntire SL, Garriga G, White J, Jacobson D, Horvitz HR (1992) Genes necessary for directed axonal elongation or fasciculation in $C$. elegans. Neuron 8:307-322.

Priess JR, Hirsh DI (1986) Caenorhabditis elegans morphogenesis: the role of the cytoskeleton in elongation of the embryo. Dev Biol 117:156-173.

Reichardt LF, Tomaselli KJ (1991) Extracellular matrix molecules and their receptors: functions in neural development. Annu Rev Neurosci 14:531-570.

Savage C, Hammelin M, Culotti JG, Coulson A, Albertson DG, Chalfie $M$ (1989) mec-7 is a beta-tubulin gene required for the production of 15-protofilament microtubules in Caenorhabditis elegans. Genes Dev 3:870-881.

Siddiqui S (1990) Mutations affecting axonal growth and guidance of motor neurons and mechanosensory neurons in the nematode Caenorhabditis elegans. Neurosci Res [Suppl] 13:171-190.

Sulston J, Horvitz HR (1977) Post-embryonic cell lineages of the nematode Caenorhabditis elegans. Dev Biol 56:110-156.

Sulston J, Schierenberg E, White JG, Thomson JN (1983) The embryonic cell lineage of the nematode Caenorhabditis elegans. Dev Biol 100:64-119.

Tessier-I avigne M, Placzek M (1991) Target attraction: are developing axons guided by chemotropism? Trends Neurosci 14:303-310.

Trent C, Tsung N, Horvitz HR (1983) Egg-laying defective mutants of the nematode Cacnorhabditis elcgans. Genetics 104:619-647.

Walthall WW, Chalfie M (1988) Cell-cell interactions in the guidance of late-developing neurons in Caenorhabditis elegans. Science 239: 643-645.

Way J, Chalfie M (1988) mec-3, a homeobox-containing gene that specifies differentiation of the touch receptor neurons in C. elegans. Cell 54:5-16.

Way JC, Chalfie M (1989) The mec-3 gene of Caenorhabditis elegans requires its own product for maintained expression and is expressed in three neuronal cell types. Genes Dev 3:1823-1833.

White JG, Southgate E, Thomson JN, Brenner S (1976) The structure of the ventral cord of Caenorhabditis elegans. Philos Trans R Soc Lond [Biol] 275:327-348.

White JG, Southgate E, Thomson JN, Brenner S (1986) The structure of the nervous system of the nematode Caenorhabditis elegans. Philos Trans R Soc Lond [Biol] 314:1-340. 